ARTICLE

https://doi.org/10.1038/s41467-019-13671-8

\title{
HDAC3 maintains oocyte meiosis arrest by repressing amphiregulin expression before the LH surge
}

Huarong Wang 1,2,7, Han Cai ${ }^{2,7}$, Xiao Wang1,3,7, Meiling Zhang4, Bingying Liu', Ziqi Chen', Tingting Yang ${ }^{1}$

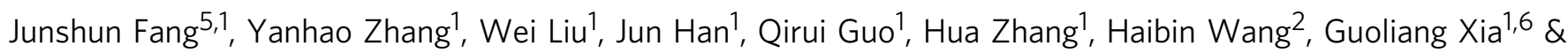
Chao Wang ${ }^{1 \star}$

It is known that granulosa cells (GCs) mediate gonadotropin-induced oocyte meiosis resumption by releasing EGF-like factors in mammals, however, the detailed molecular mechanisms remain unclear. Here, we demonstrate that luteinizing hormone (LH) surgeinduced histone deacetylase 3 (HDAC3) downregulation in GCs is essential for oocyte maturation. Before the $\mathrm{LH}$ surge, HDAC3 is highly expressed in GCs. Transcription factors, such as FOXO1, mediate recruitment of HDAC3 to the amphiregulin (Areg) promoter, which suppresses AREG expression. With the LH surge, decreased HDAC3 in GCs enables histone H3K14 acetylation and binding of the SP1 transcription factor to the Areg promoter to initiate AREG transcription and oocyte maturation. Conditional knockout of Hdac3 in granulosa cells in vivo or inhibition of HDAC3 activity in vitro promotes the maturation of oocytes independent of LH. Taking together, HDAC3 in GCs within ovarian follicles acts as a negative regulator of EGF-like growth factor expression before the LH surge.

\footnotetext{
${ }^{1}$ State Key Laboratory of Agrobiotechnology, College of Biological Sciences, China Agricultural University, 100193 Beijing, China. ${ }^{2}$ Medical College of Xiamen University, 361005 Xiamen, China. ${ }^{3}$ Beijing Advanced Innovation Center for Genomics, Biomedical Pioneering Innovation Center, College of Life Sciences, Peking University, 100871 Beijing, China. ${ }^{4}$ Shanghai Key Laboratory for Assistant Reproduction and Reproductive Genetics, Center for Reproductive Medicine, Ren Ji Hospital, School of Medicine, Shanghai Jiao Tong University, 200127 Shanghai, China. ${ }^{5}$ Reproductive Medical Center, Drum Tower Hospital Affiliated to Nanjing University Medical College, Zhongshan Road 321, 210008 Nanjing, China. ${ }^{6}$ Key Laboratory of Ministry of Education for Conservation and Utilization of Special Biological Resources in the Western China, College of Life Science, Ningxia University, 750021 Yinchuan, Ningxia, China. ${ }^{7}$ These authors contributed equally: Huarong Wang, Han Cai, Xiao Wang *email: wangcam@cau.edu.cn
} 
n mammalian follicles, somatic cells and oocytes constitute an intriguing combination that facilitates mutual signaling and chemical communication to orchestrate oocyte meiotic maturation in response to gonadotropin stimulation ${ }^{1,2}$. Fully grown oocytes are generally arrested at prophase of meiosis I by the accumulation of cyclin AMP (cAMP) produced by G-proteincoupled receptor (GPR3/12) signaling to inhibit the activity of maturation-promoting factor ${ }^{2}$. Prior to the luteinizing hormone (LH) surge, follicle-stimulating hormone (FSH) promotes the secretion of natriuretic peptide precursor type C (CNP/NPPC) from mural granulosa cells (mGCs) to maintain oocyte meiosis arrest via the regulation of cyclin GMP (cGMP) produced by cumulus cells ${ }^{3}$. cGMP enters oocytes via gap junctions to evoke meiosis arrest by inhibiting PDE3A activity, which specifically degrades CAMP $^{4,5}$.

When the LH surge occurs, LH decreases NPPC/NPR2 expression levels, thereby blocking cGMP synthesis. Simultaneously, LH stimulates mGCs to secrete epidermal growth factor (EGF)-like growth factors, especially amphiregulin (AREG), epiregulin, and beta-cellulin, which activate EGF receptor (EGFR) signaling in cumulus cells, leading to cGMP degradation in response to PDE5 activation and NPR2 inactivation and resulting in cumulus expansion and oocyte maturation. Finally, LH terminates GC proliferation and induces GC luteinization by activating extracellular signal-regulated kinase $1 / 2$ signaling ${ }^{6}$. The molecular regulatory mechanism of the sex hormone-controlled expression of NPPC and its receptor (NPR2) in response to FSH/ $\mathrm{LH}$ induction in vivo has been reported ${ }^{7,8}$. However, the signaling pathway downstream of $\mathrm{LH}$ receptor involved in regulating EGFlike growth factor expression and release has not yet been characterized.

Although epigenetic modifications are important for germ cell development ${ }^{1,9-13}$, few reports have assessed the effects of epigenetic modifications in somatic follicular cells on gonadotropininduced oocyte maturation. One exception is a study showing that the level of histone deacetylase 3 (HDAC3) was increased in GCs retrieved from polycystic ovary syndrome patients who experienced in vitro fertilization (IVF) failure compared with those from patients who experienced successful $\mathrm{IVF}^{14}$. HDAC3 belongs to the class I family of HDACs that regulates gene expression by acetylating lysine residues in histone tails ${ }^{15,16}$. Therefore, we hypothesize that HDAC3 levels in GCs may be associated with oocyte maturation quality. In addition, it remains unknown whether this association is related to gonadotropin induction.

Here we demonstrate that HDAC3 inhibits AREG expression through H3K14 deacetylation prior to the LH surge. Conditional knockout and inhibition of HDAC3 in GCs increase AREG expression and result in $\mathrm{LH}$-independent oocyte maturation in mice.

\section{Results}

HDAC3 negatively regulated oocyte maturation. To investigate the physiological role of HDACs during oocyte maturation in mice, the expression patterns of all of the class I HDACs in growing follicles induced by gonadotropins were studied. HDAC3 was the only HDAC in GCs that was upregulated by FSH but downregulated by LH in vivo and in vitro (Fig. 1a, b; Supplementary Fig. 1a-c), demonstrating its pivotal role in response to gonadotropin induction during oocyte maturation.

Then, to investigate the precise ovarian function of HDAC3, a mouse model with GC-specific deletion of Hdac3 was produced by crossing Hdac3flox/flox female mice with Foxl2-Cre $\mathrm{ER}^{\mathrm{T} 2}$ transgenic mice plus Hdac $3^{\text {flox/flox }}$ male mice, yielding Hdac3flox/flox; Foxl2-CreER ${ }^{\mathrm{T} 2}$ (Hdac3 $\left.{ }^{\mathrm{cKO}}\right)$ female mice ${ }^{17}$. Eight days after the respective intraperitoneal (i.p.) injection of tamoxifen in 18-day-old $H d a c 3^{\text {cKO }}$ female mice and Hdac $3^{\text {flox/flox }}$ female mice (as a negative control), the western blotting results showed that HDAC3 protein levels in GCs were markedly decreased in Hdac $3^{\mathrm{cKO}}$ mice compared with the control (Fig. 1c).

To clarify whether the deletion of HDAC3 in GCs correlates with oocyte maturation, pregnant mare serum gonadotropin (PMSG) was respectively injected into $H d a c 3^{\mathrm{cKO}}$ and $H d a c 3^{\text {flox/flox }}$ mice to promote follicle development 6 days after tamoxifen administration. Although Hdac3 depletion was incomplete due to limitations of the mouse model, we found that the oocytes in Hdac3-deleted follicles were premature $44 \mathrm{~h}$ after PMSG injection, while the

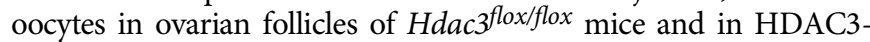
positive follicles of $\mathrm{Hdac}^{\mathrm{cKO}}$ mice, similar to the oocytes in wildtype (WT) mice and the Foxl2-CreER ${ }^{\mathrm{T} 2}$ transgene but not carrying the floxed allele mice, were still at the germinal vesicle (GV) stage (Fig. 1d, e; Supplementary Fig. 2a-c). These results indicated that Hdac3 deletion results in premature oocyte maturation before the LH surge begins.

To examine whether Hdac3 depletion affects ovarian follicle survival and follicular atresia, which probably result in oocyte GV breakdown (GVBD), we performed quantitative polymerase chain reaction ( $\mathrm{qPCR}$ ) to determine the $B c l 2 / \mathrm{Bax}$ ratio and terminal deoxynucleotidyl transferase-mediated dUTP-fluorescein nick end labeling (TUNEL) assays to evaluate GC survival. The results showed that the $B c l 2 / B a x$ ratio was normal in $H d a c 3^{\mathrm{CKO}}$ mouse ovarian GCs (Supplementary Fig. 2d). Similarly, TUNEL assays showed that oocyte GVBD in Hdac3 knockout ovarian follicles was not caused by GC apoptosis or follicular atresia (Supplementary Fig. 2e). Therefore, the GC-specific knockout of Hdac3 did not affect the development or survival of GCs and follicles. Furthermore, LH can terminate ovarian GC proliferation, and we found that Hdac3 depletion also prohibited GC proliferation, as indicated by proliferating cell nuclear antigen staining (Supplementary Fig. 2f). These results indicated that Hdac3 depletion in ovarian GCs probably mimics the effects of $\mathrm{LH}$ on oocyte maturation and GC proliferation.

HDAC3 repressed AREG expression in GCs before the LH surge. Given that NPPC and EGF-like growth factors are the two types of molecules secreted by GCs that are critical in the regulation of oocyte meiosis progression in response to gonadotropin induction ${ }^{3,6}$, two possible pathways may be responsible for the effects of Hdac3 deletion on oocyte maturation. Specifically, Hdac3 deletion in GCs may either downregulate NPPC expression to relieve oocytes from the inhibitory environment or upregulate EGF-like growth factors to re-initiate oocyte meiosis. Interestingly, the mRNA and protein levels of only AREG, one of the most important EGF-like growth factors that responds to $\mathrm{LH}$ induction, were significantly increased in the ovaries of Hdac $3 \mathrm{cKO}$ mice $40 \mathrm{~h}$ after PMSG induction (Fig. 2a, b; Supplementary Fig. 3a). These findings indicate that AREG likely mediates HDAC3 action in oocyte maturation.

To confirm the HDAC3-mediated regulation of AREG expression, follicle cultures in vitro were treated with HDACi $4 \mathrm{~b}$, a novel, synthetic, specific inhibitor of $\mathrm{HDAC} 1 / 3^{18}$. Consistent with the effects of Hdac3 knockout in vivo, AREG protein and mRNA levels were significantly elevated by HDACi 4b (Fig. 2c, d). Moreover, cumulus expansion and oocyte meiosis resumption were induced by HDACi 4 b, while these effects were blocked by the EGFR inhibitor AG1478, confirming the role of AREG in the effects of HDAC3 on oocyte maturation (Fig. 2e, f). Furthermore, chromatin immunoprecipitation (ChIP) analysis showed that, before the LH surge, more HDAC3 protein was recruited to the Areg promoter, while $3 \mathrm{~h}$ after human chorionic gonadotropin 
a

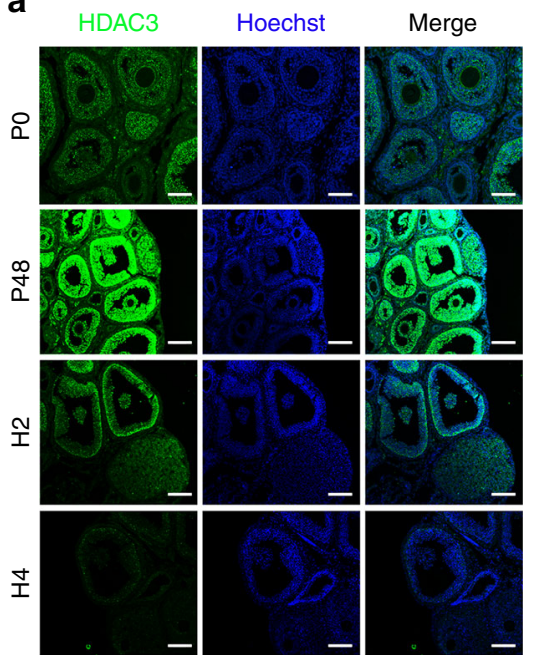

b
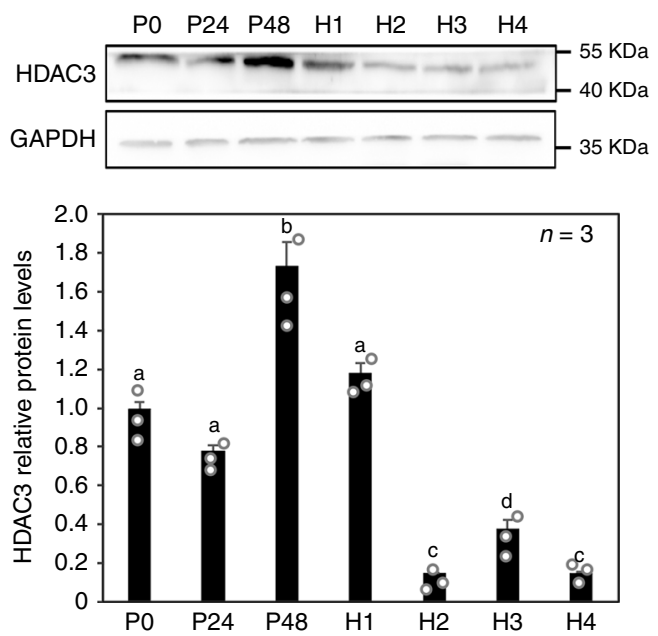

C
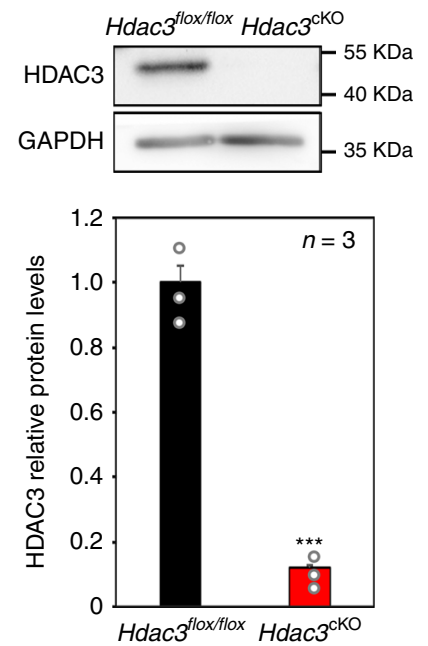

d

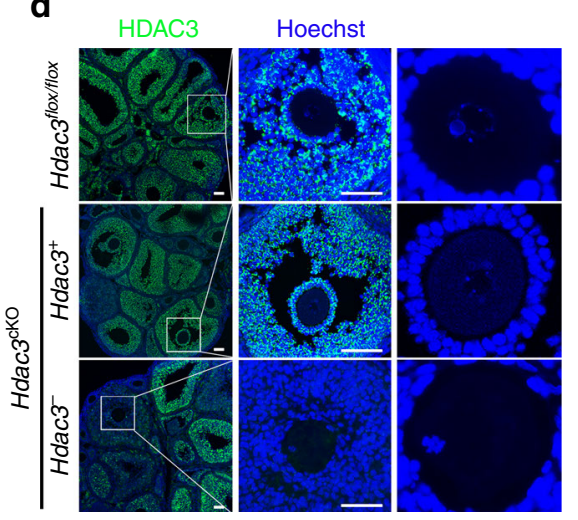

e

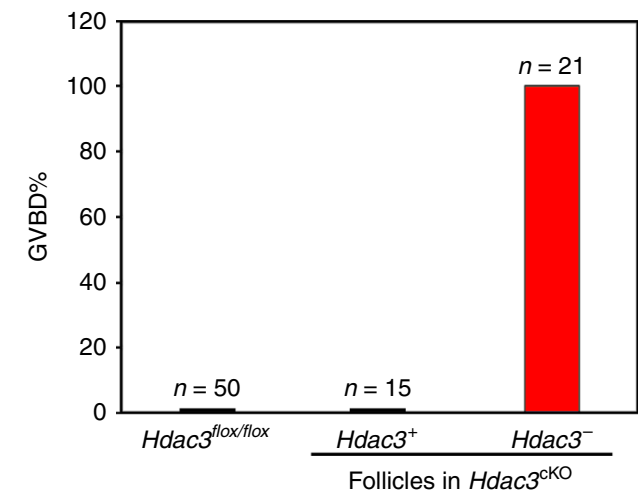

Fig. 1 HDAC3 negatively regulated oocyte maturation. a HDAC3 (green) levels in GCs decreased dramatically in response to hCG stimulation. Nuclei were dyed with Hoechst (blue). Scale bar $=100 \mu \mathrm{m} . n=3$ biologically independent experiments. b HDAC3 protein levels were upregulated by PMSG and downregulated by hCG in a time-dependent manner. P48: $48 \mathrm{~h}$ after PMSG treatment, equivalent to $48 \mathrm{~h}$ after the FSH surge. H1-H4: 1-4 $\mathrm{h}$ after $\mathrm{hCG}$ treatment, equivalent to $1-4 \mathrm{~h}$ after the LH surge. $n=3$ biologically independent experiments. Data are presented as mean \pm SEM, and different letters (a-d) indicate significant differences among groups (two-sided ANOVA test), $P(a, b)<0.05, P(a, c)<0.01, P(a, d)<0.01, P(b, c)<0.001, P(b, d)<0.01$, $P(c, d)<0.05$. c Hdac3 knockout efficiency was examined and quantified by western blotting analysis. $n=3$ biologically independent experiments. Data are presented as mean $\pm \mathrm{SEM}$. Asterisks $\left(^{\star}\right)$ indicate significant differences at ${ }^{\star \star \star} P=0.00007$ determined by the two-sided $t$ test. $\mathbf{d}$ HDAC3 (green) immunostaining in the different mouse models revealed oocyte maturation independent of $\mathrm{LH}$ in follicles lacking Hdac3 expression (Hdac3-) in GCs, whereas oocytes in $\mathrm{Hdac3}$-positive $\left(\mathrm{Hdac}^{+}\right)$follicles remained in the GV stage, similar to the situation in $\mathrm{Hdac} 3^{\text {flox/flox }}$ mice. Scale bar $=100 \mu \mathrm{m}$. Nuclear DNA in indicated in blue (Hoechst). e Oocyte maturation (GVBD) rates in follicles with or without HDAC3 expression in the different mouse genotypes. Source data are provided as a Source Data file.

(hCG) treatment (equivalent to $3 \mathrm{~h}$ after the LH surge), HDAC3 recruitment to the Areg promoter was significantly decreased (Fig. 2g). HDAC1 was unable to bind the Areg promoter (Supplementary Fig. 3b). Therefore, we concluded that HDAC3 can specifically bind to the Areg promoter region $(-343,-150)$ before the LH surge.

Furthermore, cumulus cells in cumulus oocyte complexes (COCs) were reported to express AREG upon indirect induction by the LH surge in vivo and upon direct induction by FSH in vitro during oocyte maturation ${ }^{19,20}$, suggesting that AREG expression in cumulus cells is also essential for oocyte maturation. To examine whether HDAC3 regulates AREG expression in cumulus cells, we examined Areg expression levels after mouse COCs were cultured with or without HDACi $4 \mathrm{~b}$ in vitro in NPPC-M199 medium. The results showed that HDACi $4 \mathrm{~b}$ was able to promote oocyte maturation in COCs by directly inducing AREG expression in cumulus cells (Supplementary Fig. 4). Collectively, the data indicated that HDAC3 is a negative regulator of AREG expression that is essential for gonadotropin-induced oocyte maturation.

It is well known that cGMP produced by NPR2 is essential for oocyte meiosis arrest, which affects upon diffusion via gap junctions ${ }^{2}$. AREG activates EGFR signaling in cumulus cells, which suppresses the guanylyl cyclase activity of NPR2 and activates PDE5 activity to decrease cGMP levels ${ }^{2,21}$. To examine whether the HDAC3 inhibitor induces oocyte maturation by changing cGMP levels in cumulus cells, we cultured COCs with 8-Br-cGMP alone or in combination with HDACi 4b in M199 medium. The results showed that, while most of the oocytes in the control group resumed meiosis, the oocytes in the group treated with 8-Br-cGMP alone arrested at the GV stage; however, the oocytes in the group treated with HDACi $4 \mathrm{~b}$ and 8 -Br-cGMP were unable to resume meiosis, with most remaining at the GV stage (Fig. 2h). Therefore, we concluded that HDAC3 deficiency induced oocyte maturation by regulating the levels of cGMP, 
a

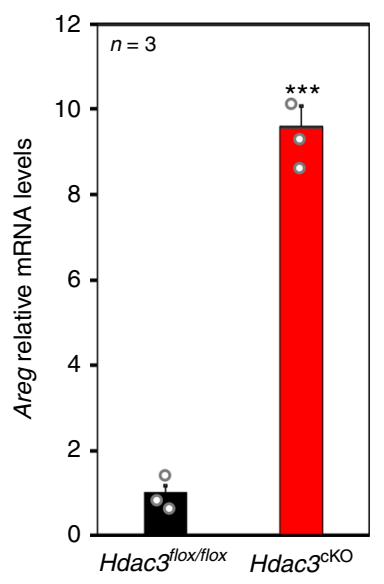

b
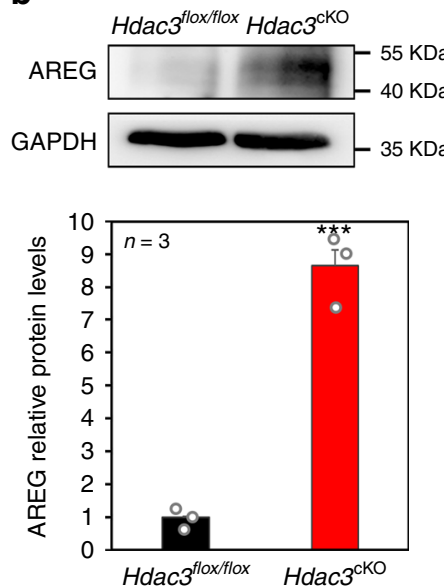

c

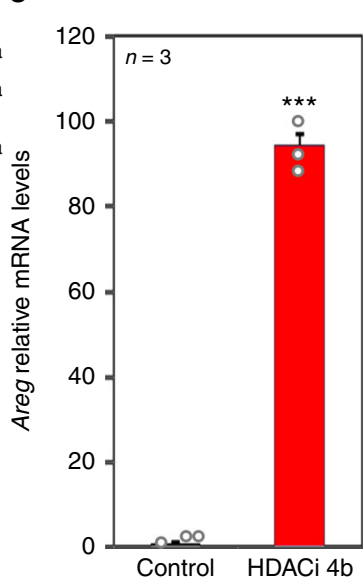

d
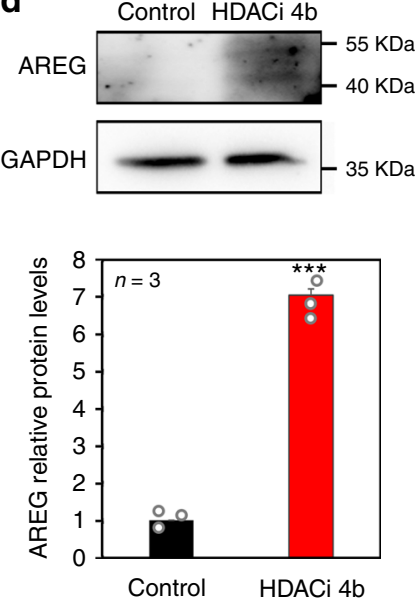

e

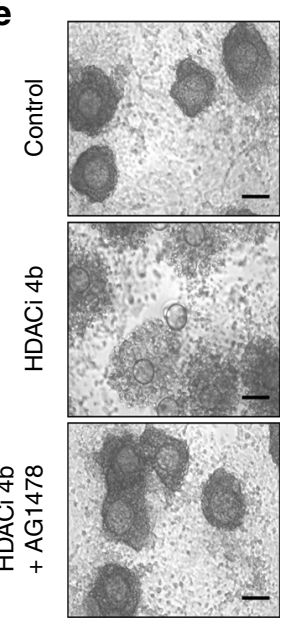

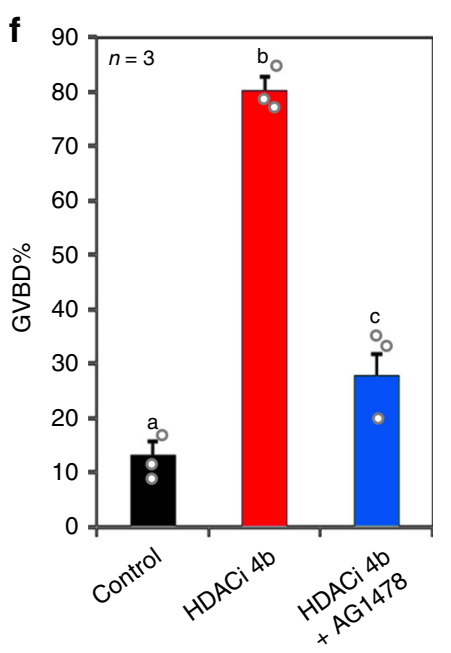

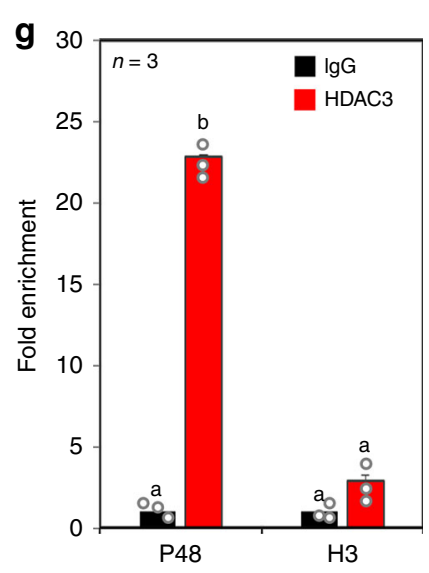

h

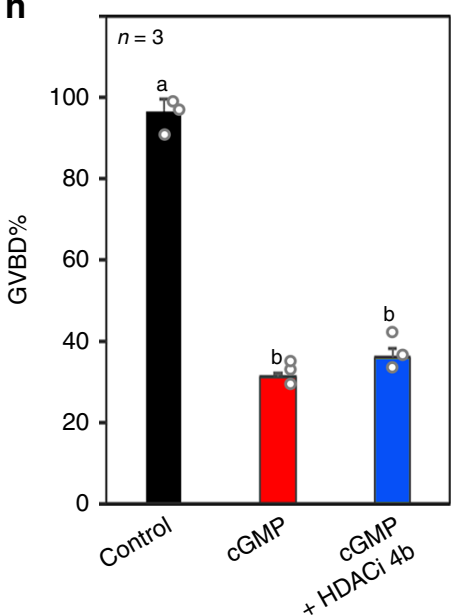

j

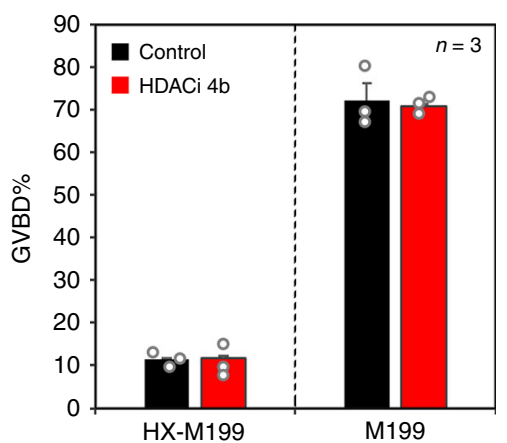

i

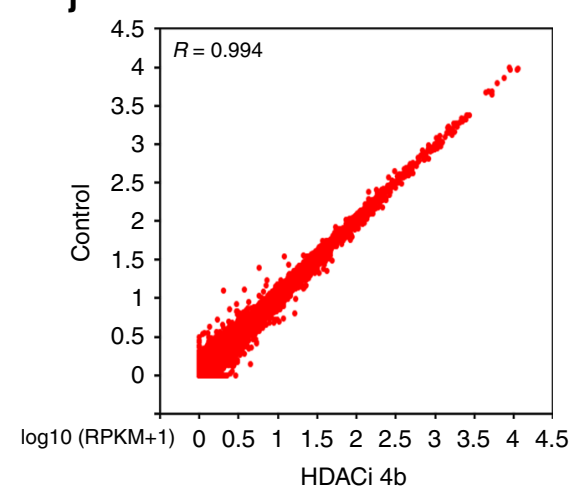

$\mathbf{k}$

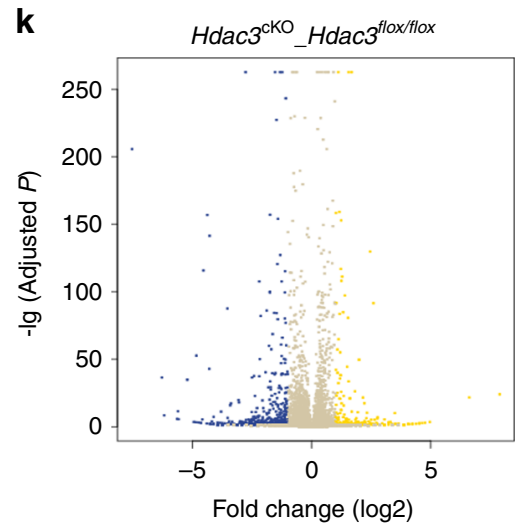

which is well known to diffuse from cumulus cells to oocytes through gap junctions ${ }^{2}$.

An important question is whether HDACi $4 \mathrm{~b}$ directly affects denuded oocyte maturation and developmental competence. Therefore, the effects of HDACi $4 \mathrm{~b}$ on denuded oocytes were examined in HX-M199 and M199 media. In HX-M199 medium, most of the oocytes in the control and HDACi 4b-treated groups were arrested in the GV stage (Fig. 2i; Supplementary Fig. 5). In M199 medium, most of the oocytes in the control and HDACi 4 b-treated groups resumed meiosis (Fig. 2i; Supplementary Fig. 5). These results demonstrated that HDACi $4 \mathrm{~b}$ has no direct effect on oocyte meiotic maturation.

Furthermore, to detect whether HDACi $4 \mathrm{~b}$ affects developmental competence, we performed transcriptome analysis of all the mature oocytes in the control and HDACi $4 \mathrm{~b}$-treated groups. The correlation coefficient between global transcripts in the control and HDACi 4b-treated groups was $>0.99$, and no differentially expressed genes were found (Fig. $2 \mathrm{j}$ and Supplementary Data 1), indicating that HDACi 4 b has no effect on oocyte developmental competence.

Although HDAC3 and HDACi 4b controlled oocyte maturation by regulating AREG expression was proved in this study, other oocyte maturation-related factors regulated by HDAC3 remained unidentified. Therefore, we performed RNA-seq analysis of ovarian GCs from Hdac $3^{\mathrm{cKO}}$ and Hdac3 flox/flox mice and of GCs treated with HDACi $4 \mathrm{~b}$ or control. Compared with ovarian GCs from $\mathrm{Hdac} 3^{\text {flox/flox }}$ mice, those from $\mathrm{Hdac} 3^{\mathrm{cKO}}$ mice showed the differential expression of as many as 233 transcripts, 
Fig. 2 HDAC3 repressed AREG expression in GCs before the LH surge. a Quantitative real-time PCR revealed Areg expression in Hdac 3 flox/flox and Hdac $^{\mathrm{CKO}}$ GCs after PMSG treatment. Data are presented as mean \pm SEM. Asterisks $\left(^{\star}\right)$ indicate significant differences at ${ }^{\star \star \star} P=0.000003$ determined by the two-sided $t$ test. b AREG levels in Hdac3flox/flox and Hdac3 ${ }^{K K O}$ GCs were quantified by western blotting analysis. $n=3$ biologically independent experiments. Data are presented as mean \pm SEM. Asterisks $\left(^{\star}\right)$ indicate significant differences at ${ }^{\star \star \star} P=0.0009$ determined by the two-sided $t$ test. c Quantitative real-time PCR showed that inhibiting HDAC3 increased Areg expression in GCs in vitro. HDACi 4b, an HDAC3 inhibitor. Data are presented as mean \pm SEM. Asterisks $\left({ }^{\star}\right)$ indicate significant differences at ${ }^{\star \star \star} P=5.15 \mathrm{E}-5$ determined by the two-sided $t$ test. $\mathbf{d}$ AREG levels in control and HDACi 4 b-treated GCs were quantified by western blotting analysis. $n=3$ biologically independent experiments. Data are presented as mean \pm SEM. Asterisks $\left({ }^{\star}\right)$ indicate significant differences at ${ }^{\star \star \star} P=3.27 \mathrm{E}-5$ determined by the two-sided $t$ test. e Representative images demonstrate the effect of GC-derived HDAC3 on cumulus cell expansion within in vitro cultured COCs. Scale bar $=100 \mu \mathrm{m} . n=3$ biologically independent experiments. f Oocyte maturation (GVBD) rates were significantly improved by HDACi 4b, and this effect was reversed by AG1478. AG1478, EGFR inhibitor. $n=3$ biologically independent experiments. Data are presented as mean \pm SEM, and different letters (a-c) indicate significant differences among groups (two-sided ANOVA and Holm-Šidák test), $P(a, b)<0.001, P(a, c)<0.05, P(b, c)<0.001$. $\mathbf{g}$ ChIP-qPCR analysis of HDAC3 at the Areg promoter $(-343$ to -150$)$ before and after the LH surge. $n=3$ biologically independent experiments. Data are presented as mean \pm SEM, and different letters (a-b) indicate significant differences among groups (twosided ANOVA and Holm-Šidák test), $P(a, b)<0.001$. h HDACi 4b was unable to rescue 8-Br-cGMP-mediated inhibition of oocyte maturation (GVBD). Data are presented as mean \pm SEM, and different letters ( $a-b)$ indicate significant differences among groups (two-sided ANOVA and Holm-Šidák test), $P$ ( $a, b$ ) < 0.001 . i HDACi $4 \mathrm{~b}$ had no direct effect on denuded oocyte meiotic maturation. $\mathbf{j}$ Scatter plot comparison of oocyte transcripts between the control and HDAC3 inhibitor groups revealed that HDACi $4 \mathrm{~b}$ had no effect on oocyte developmental competence. Pearson statistical test used for statistical analysis. $\mathbf{k}$ Volcano plot of transcriptomic analysis of GCs isolated from Hdac flox/flox and Hdac3cKO ovaries. Source data are provided as a Source Data file.

with 78 upregulated transcripts, including Areg, and 155 downregulated transcripts (Fig. 2k and Supplementary Data 2). The genes with the most pronounced changes in expression are shown in Supplementary Fig. 6a. Impressively, more general transcriptome changes were detected in GCs treated with HDACi $4 \mathrm{~b}$ in vitro than in those treated with the control: 2105 transcripts were upregulated and 1301 transcripts were downregulated (Supplementary Fig. 6c and Supplementary Data 3). The Gene Ontology (GO) terms associated with the altered transcripts were also analyzed (Supplementary Fig. 6b, d).

H3K14ac mediated HDAC3 and LH action regarding Areg expression. It is well known that HDAC3 packages chromatin into repressed domains via its HDAC activity ${ }^{22,23}$. To identify which histone lysine residues are regulated by HDAC3 in GCs, most of the histone acetylation modifications in GCs during LH/ hCG induction were assessed (Supplementary Fig. 7a, b). The results showed that histone 3 lysine 14 acetylation (H3K14ac) was markedly increased by LH/hCG induction in vivo and in vitro (Fig. 3a, b; Supplementary Fig. 7c, d). ChIP analysis demonstrated that H3K14ac levels at the Areg promoter were substantially increased in GCs induced by LH/hCG (Fig. 3c), suggesting that H3K14ac probably mediates the actions of $\mathrm{LH} / \mathrm{hCG}$ on target gene expression.

Furthermore, to elucidate whether HDAC3 inhibits AREG expression by repressing H3K14ac, GCs from HDAC3 knockout mouse ovaries or HDACi $4 \mathrm{~b}$-treated follicles were examined. H3K14ac levels were significantly increased in ovarian GCs from $\mathrm{Hdac}{ }^{\mathrm{CKO}}$ mice compared with $\mathrm{Hdac} 3^{\text {flox/flox }}$ mice (Fig. 3d). Furthermore, ChIP analysis revealed that H3K14ac levels at the Areg promoter were obviously increased in GCs from Hdac3 ${ }^{\mathrm{CKO}}$ mouse ovaries compared with Hdac $3^{\text {flox/flox }}$ mouse ovaries (Fig. 3e). Correspondingly, H3K14ac levels were much higher in HDACi 4b-treated GCs than in control GCs (Fig. 3f). ChIP analysis showed that HDACi 4b increased H3K14ac levels at the Areg promoter in GCs in vitro (Fig. 3g). Therefore, H3K14ac is key for the regulation of AREG expression by both LH and HDAC3. The results unanimously confirmed our hypothesis that HDAC3 decreases H3K14ac levels in vivo and in vitro before $\mathrm{LH}$ induction.

FOXO1 potentially recruited HDAC3 to the Areg promoter. Among the class I HDAC family, HDAC3 is the only member that functions through interacting with NCoR1/2 and TBL1X/ TBL1XR, forming the core of the NCoR transcriptional repressor complex through its deacetylase activation domain ${ }^{24}$. In line with this finding, we performed coimmunoprecipitation (Co-IP) of HDAC3 coupled with mass spectrometry (MS) to identify factors that bind HDAC3 to repress Areg expression. The core members of the NCoR complex, NCoR1, NCoR2, and TBL1X, were all identified in the HDAC3 antibody group compared with the immunoglobulin G (IgG) group (Fig. 4a; Supplementary Data 4 and 5).

Another important unknown is the transcription factor that recruits the HDAC3-NCoR complex to the Areg promoter. We performed DNA pulldown coupled with MS analysis and searched for predicted transcription factor-binding sites using the JASPAR database. By comparing the proteins captured by Areg promoter DNA with the transcription factor-binding data predicted by JASPAR, four candidate transcription factors, namely, SP1, FOXO1, GATA4, and Myod1, were identified (Fig. 4b; Supplementary Data 6 and 7). However, none of these transcription factors were identified by HDAC3 Co-IP (Fig. 4a; Supplementary Data 4 and 5), indicating that they potentially interact with the HDAC3-NCoR complex rather than with HDAC3 directly. Therefore, we performed cross-linking Co-IP followed by MS to examine all of the proteins directly or indirectly interacting with HDAC3 on chromatin ${ }^{25}$. Western blotting analysis of cross-linking Co-IP against HDAC3 showed that numerous HDAC3 complexes were successfully captured in the HDAC3 IP group compared to the negative IgG group (Fig. 4c). As expected, MS data analysis identified many transcription factors among the HDAC3 interactors on chromatin, including the enrichment of NCoR complex components (Supplementary Data 8). However, only FOXO1, GATA4, and Myod1, but not SP1, were found among the HDAC3 interactors, indicating that these three transcription factors potentially interact with the HDAC3 complex at the Areg promoter (Fig. 4b; Supplementary data 8). Furthermore, the interaction between FOXO1 and HDAC3 was confirmed by reverse cross-linking CoIP coupled with western blotting (Fig. 4d). Therefore, we concluded that one or more of the FOXO1, GATA4, and Myod1 transcription factors are potentially recruited by $\mathrm{HDAC} 3$ to the Areg promoter before the LH surge.

HDAC3 and LH affected Areg expression by regulating SP1 recruitment to the Areg promoter. Histone acetylation induces gene expression by causing chromatin unfolding and increasing transcription factor access ${ }^{15}$; thus we aimed to identify the transcription factors that bind to the Areg promoter and initiate 
a
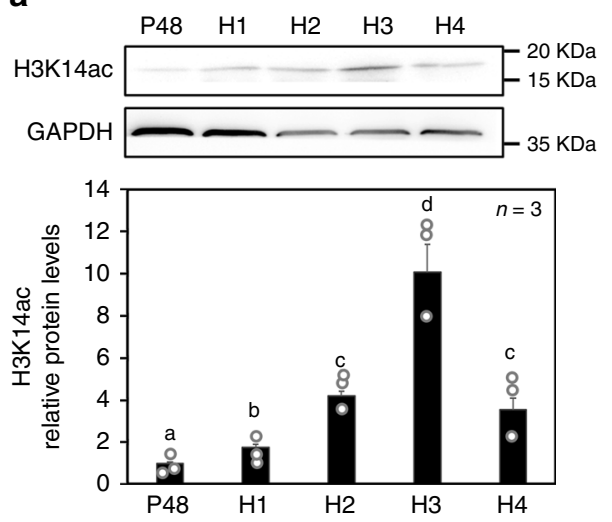

b

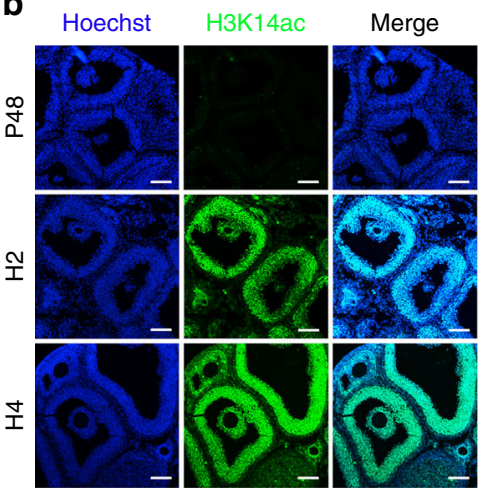

C

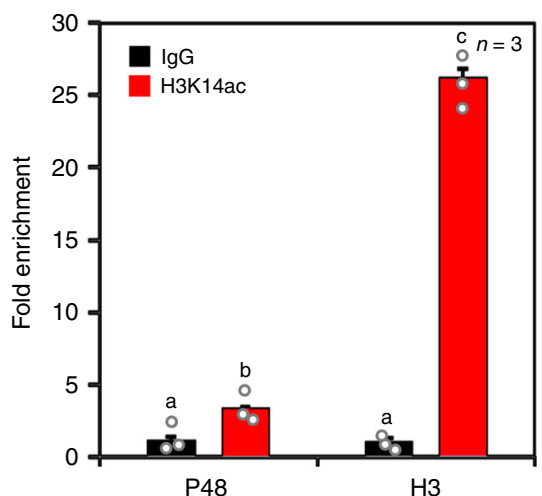

e
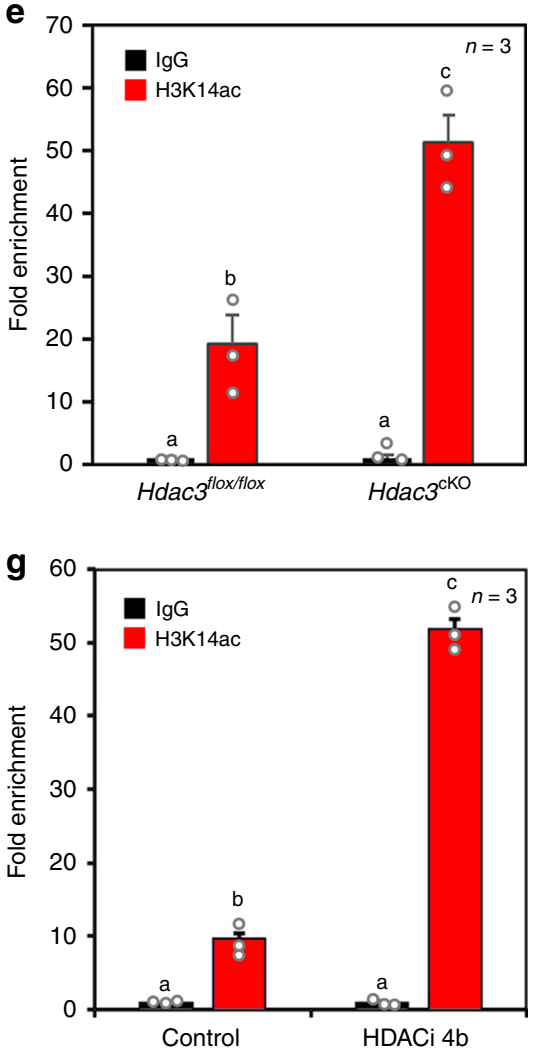

Fig. 3 H3K14ac mediated the effects of HDAC3 and LH on Areg expression. a H3K14ac levels were increased by hCG in a time-dependent manner. $n=3$ biologically independent experiments. Data are presented as mean $\pm \mathrm{SEM}$, and different letters (a-d) indicate significant differences among groups (twosided ANOVA and Holm-Šidák test), $P(a, b)<0.01, P(a, c)<0.01, P(a, d)<0.001, P(b, c)<0.05, P(b, d)<0.01, P(c, d)<0.05$. b Immunostaining revealed that LH increased H3K14ac (green) levels in GCs in vivo. Nuclei were dyed with Hoechst (blue). Scale bar $=100 \mu \mathrm{m}$. $n=3$ biologically independent experiments. c ChIP-qPCR analysis of H3K14ac at the Areg promoter ( -343 to -150 ) before and after the LH surge. P48: $48 \mathrm{~h}$ after PMSG treatment. $\mathrm{H} 1-\mathrm{H} 4: 1-4 \mathrm{~h}$ after $\mathrm{hCG}$ treatment. Data are presented as mean $\pm \mathrm{SEM}$, and different letters (a-c) indicate significant differences among groups (two-sided ANOVA and Holm-Šidák test), $P(a, b)<0.01, P(a, c)<0.001, P(b, c)<0.001$. d H3K14ac levels in Hdac3flox/flox and Hdac3cKO GCs were quantified by western blotting analysis. $n=3$ biologically independent experiments. Data are presented as mean \pm SEM. Asterisks (*) indicate significant differences at ${ }^{\star \star \star} P<0.001$ determined by the two-sided $t$ test. e ChIP-qPCR analysis of H3K14ac at the Areg promoter in Hdac3flox/flox and Hdac3cKO GCs. Data are presented as mean \pm SEM, and different letters (a-c) indicate significant differences among groups (two-sided ANOVA and Holm-Šidák test), $P(a, b)<0.001, P(a, c)<0.001, P(b, c)<0.05$. f Western blotting analysis showed that HDACi 4b (HDAC3 inhibitor) substantially increased H3K14ac levels. $n=3$ biologically independent experiments. Data are presented as mean \pm SEM. Asterisks $\left({ }^{\star}\right)$ indicate significant differences at ${ }^{* \star} P<0.001$ determined by the two-sided $t$ test. $\mathbf{g}$ ChIP-qPCR analysis of H3K14ac at the Areg promoter in control and HDACi 4b-treated GCs. Data are presented as mean $\pm \mathrm{SEM}$, and different letters $(\mathrm{a}-\mathrm{c})$ indicate significant differences among groups (two-sided ANOVA and Holm-Šidák test), $P(a, b)<0.001, P(a, c)<$ $0.001, P(b, c)<0.001$. Source data are provided as a Source Data file. 
a

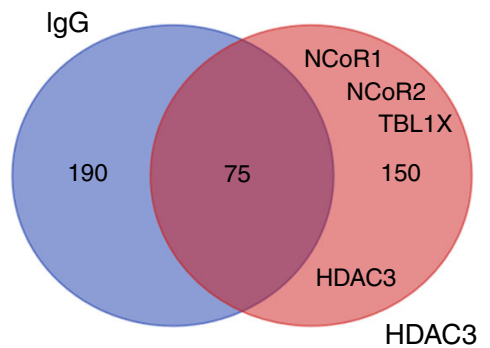

C

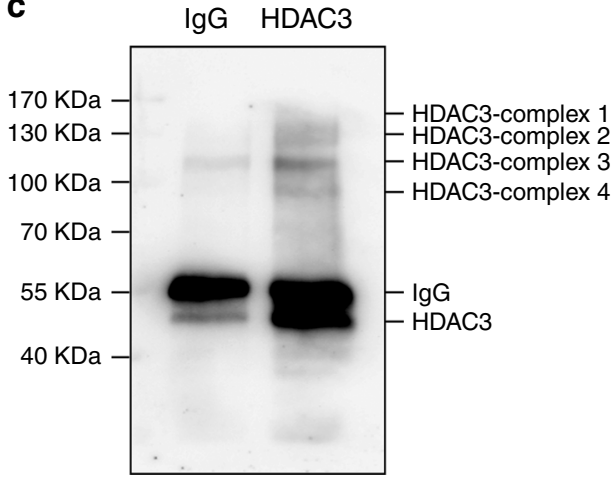

b

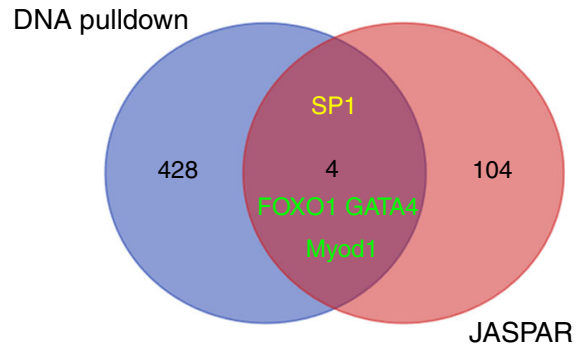

d

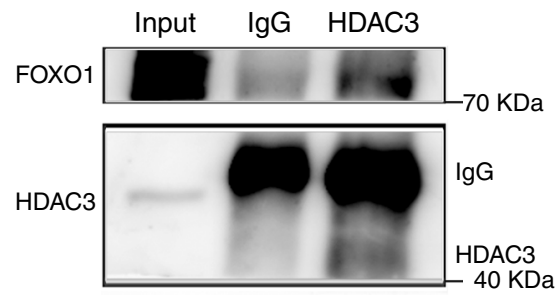

Fig. 4 FOX01 potentially recruited HDAC3 to the Areg promoter. a Venn diagram of HDAC3 partners between the HDAC3-IP group and the negative IgG group revealed that HDAC3 could interact with the core members of the NCoR complex. $\mathbf{b}$ Venn diagram of potential transcription factors that bind the Areg promoter between the DNA pulldown data and JASPAR data. Among these transcription factors, FOXO1, GATA4, and Myod1 (green), but not SP1 (yellow), were identified as HDAC3 interactors. c Cross-linking Co-IP coupled with western blotting analysis of HDAC3 showed the captured HDAC3 complexes at the chromatin. $n=3$ biologically independent experiments. d Reverse cross-linking Co-IP coupled with western blotting analysis of HDAC3 demonstrated the interaction between HDAC3 and FOXO1. $n=3$ biologically independent experiments. Source data are provided as a Source Data file.

transcription upon removal of HDAC3. We found that AREG expression induced by HDAC3 inhibition was reversed by mithramycin A (MIT), a specific inhibitor of the transcription factor SP1 (Fig. 5a), suggesting that SP1 is involved in Areg transcription after HDAC3 downregulation. Although HDACi 4b and Hdac3 knockout had no effect on SP1 expression in vitro (Fig. 5b) or in vivo (Fig. 5d), respectively, ChIP analysis revealed increased SP1 protein recruitment to the HDAC3-binding site on the Areg promoter in HDACi 4b-treated GCs compared with control cells (Fig. 5c). Correspondingly, Hdac3 deletion in ovarian GCs significantly increased SP1 occupancy at the Areg promoter (Fig. 5e). Collectively, the loss of HDAC3 and the acetylation of H3K14 promoted SP1 binding to the Areg promoter.

To investigate the role of SP1 under more physiological conditions, we examined the effect of SP1 on LH-induced AREG expression. MIT notably reversed the high AREG levels induced by LH (Fig. 5f), suggesting that SP1 is involved in Areg transcription induced by LH. Consistently, SP1 protein levels in GCs were unaffected by LH during oocyte maturation (Fig. 5g), whereas ChIP analysis revealed that LH strongly promoted SP1 recruitment to the HDAC3-binding site on the Areg promoter (Fig. 5h). Taken together, these data indicated that $\mathrm{LH}$ induces H3K14ac and increases SP1 access to the Areg promoter.

The HDAC3 inhibitor improved mouse oocyte maturation and developmental competence. In light of our findings demonstrating that the inhibition of GC-derived HDAC3 induces oocyte maturation independent of $\mathrm{LH}$ action, we assessed whether HDAC3 is a potential target to improve the developmental capacity of oocytes undergoing in vitro maturation (IVM) independent of LH. Briefly, HDACi $4 \mathrm{~b}$ was applied to cultured mouse COCs with mGC feeders, which is known as the HDACi $4 \mathrm{~b}-\mathrm{GC}$ feeder system. Although the oocyte GVBD rate of COCs was similar between the control and HDACi 4 b groups (93\% vs. $94 \%$, respectively; $P>0.05$ ) (Fig. $6 \mathrm{a}$ ), the ratio of metaphase II (MII) oocytes was 1.9 -fold higher in the HDACi $4 \mathrm{~b}$ group than in the control group ( $85 \%$ vs. $45 \%, P<0.01$ ) (Fig. $6 \mathrm{~b}$ ), demonstrating that our system promoted the GV to MII transition in oocytes.

Then oocyte developmental capacity was further evaluated. In brief, all the MII-stage oocytes obtained from the IVM system were used for IVF. After 4 days of in vitro culture (IVC) of fertilized eggs, up to 2.2-fold more blastocysts were obtained from the HDACi 4 b group than from the control group (Fig. $6 c, d$ ). The embryo transfer assay demonstrated that the blastocysts in the HDACi $4 \mathrm{~b}$ group could undergo full pregnancy and even produce slightly more pups, although the difference in pup number was not statistically significant (Fig. 6e, f). Interestingly, the body weights of the pups in the control and HDACi $4 \mathrm{~b}$ groups at 3 days after birth were similar (Fig. 6g). Collectively, by targeting HDAC3, our IVM system promotes oocyte nuclear and cytoplasmic development competence independent of $\mathrm{LH}$.

\section{Discussion}

Physiologically, the final stage of oocyte maturation requires close cooperation between oocytes and GCs within the follicles ${ }^{25}$. Consistent with this finding, $\mathrm{LH}$ response failure leads to failed oocyte maturation and ovulation, and this response is related to abnormal gene expression in $\mathrm{GCs}^{1,9,26-29}$. It is therefore reasonable to hypothesize that an essential inhibitory mechanism in GCs may exist that restrains gene expression important for resuming oocyte maturation before the $\mathrm{LH}$ induction. The inability to repress the expression of these genes leads to the gonadotropin-independent resumption of meiosis, which interrupts the synchrony between oocyte maturation and ovulation and compromises female fertility ${ }^{3}$. Surprisingly, this study revealed an epigenetic modification mechanism in GCs that 
a

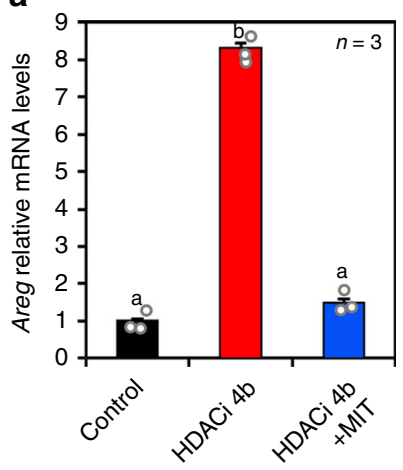

d

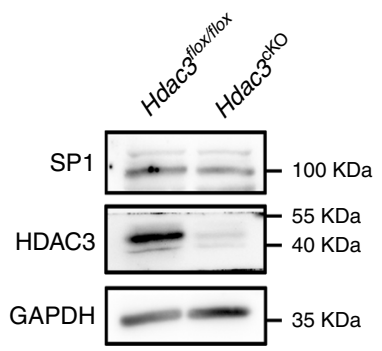

b

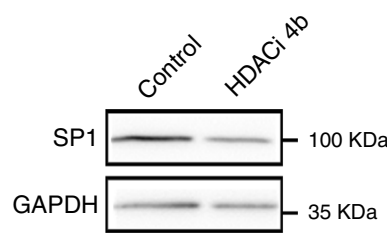

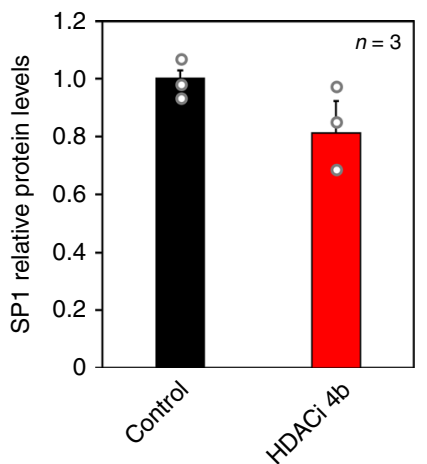

C
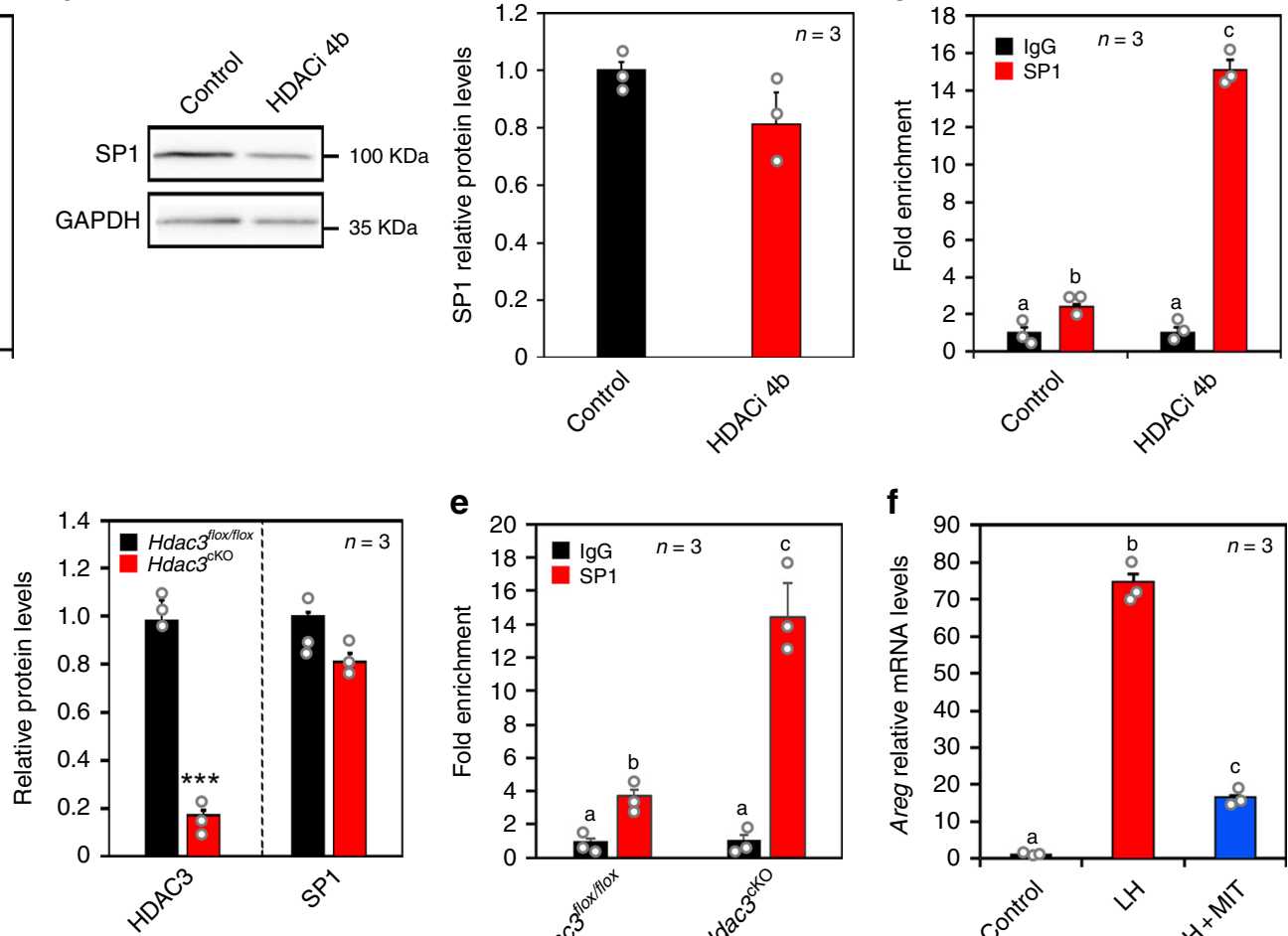

e

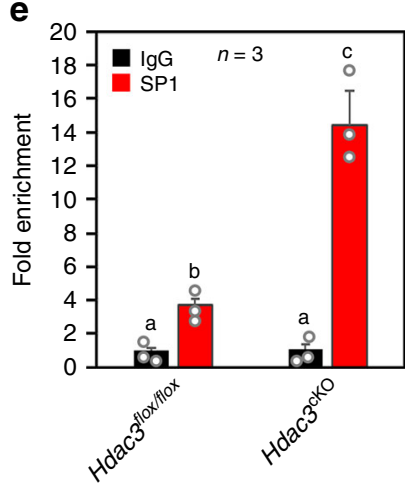

f

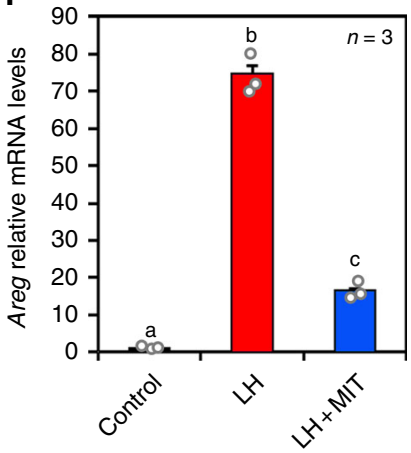

h

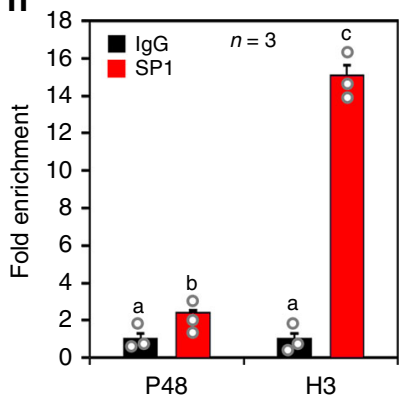

Fig. 5 HDAC3 and LH affected Areg expression by regulating SP1 recruitment to the Areg promoter. a Inhibition of SP1 reversed the HDACi 4b-mediated induction of Areg expression. MIT, SP1 inhibitor. Data are presented as mean \pm SEM, and different letters (a-b) indicate significant differences among groups (two-sided ANOVA and Holm-Šidák test), $P(a, b)<0.001$. b Western blotting analysis revealed that inhibition of HDAC3 did not affect SP1 expression. HDACi 4b, HDAC3 inhibitor. $n=3$ biologically independent experiments. Data are presented as mean \pm SEM. c ChIP-qPCR analysis of SP1 at the Areg promoter ( -343 to -150 ) in control and HDACi $4 b$-treated GCs. Data are presented as mean \pm SEM, and different letters (a-c) indicate significant differences among groups (two-sided ANOVA and Holm-Šidák test), $P(a, b)<0.05, P(a, c)<0.001, P(b, c)<0.001$. d SP1 levels in Hdac3flox/flox and Hdac3CKO GCs were quantified by western blotting analysis. $n=3$ biologically independent experiments. Data are presented as mean \pm SEM. Asterisks $\left({ }^{\star}\right)$ indicate significant differences at ${ }^{\star \star \star} P<0.001$ determined by the two-sided $t$ test. e ChIP-qPCR analysis of SP1 at the Areg promoter in Hdac3flox/flox and Hdac3 ${ }^{c K O}$ GCs. Data are presented as mean \pm SEM, and different letters (a-c) indicate significant differences among groups (two-sided ANOVA and Holm-Šidák test), $P(a, b)<0.05, P(a, c)<0.001, P(b, c)<0.001$. $\mathbf{f}$ Inhibition of SP1 reversed the LH-mediated induction of Areg expression. Data are presented as mean \pm SEM, and different letters (a-c) indicate significant differences among groups (two-sided ANOVA and Holm-Šidák test), $P(a, b)<0.001, P(a, c)<0.001, P(b, c)<0.001$. $\mathbf{g}$ Western blotting analysis revealed that LH did not affect the SP1 expression in vivo. $n=3$ biologically independent experiments. Data are presented as mean \pm SEM. $\mathbf{h}$ ChIP-qPCR analysis of SP1 at the Areg promoter before and after the LH surge. P48: $48 \mathrm{~h}$ after PMSG treatment. $\mathrm{H} 1-\mathrm{H} 4: 1-4 \mathrm{~h}$ after $\mathrm{hCG}$ treatment. Data are presented as mean $\pm \mathrm{SEM}$, and different letters (a-c) indicate significant differences among groups (two-sided ANOVA and Holm-Šidák test), $P(a, b)<0.05, P(a, c)<0.001, P(b, c)<0.001$. Source data are provided as a Source Data file.

histone acetylation by HDAC3 is essential for gonadotropininduced oocyte maturation (Supplementary Fig. 8).

EGF-like growth factors mediate the effect of LH on oocyte maturation ${ }^{30}$. Among these factors, AREG is the most abundant and important protein ${ }^{26}$. The Areg knockout mouse has severely impaired fertility ${ }^{31}$, and AREG levels in follicular fluid are positively correlated with the number of available embryos in humans ${ }^{32}$. However, given the significant differences in bioactivity between endogenous and recombinant AREG, the concentration of recombinant AREG necessary to promote cumulus expansion and oocyte maturation in vitro is approximately 20-fold higher than that detected in follicular fluid ${ }^{26}$. Therefore, employing GC-secreted AREG to improve oocyte maturation in vitro may be a more efficient and physiological strategy. Here AREG expression in both GCs and cumulus cells is essential for oocyte maturation under physiological conditions ${ }^{19}$. HDAC3 regulates AREG expression in both GCs and cumulus cells (Fig. 2a-d; Supplementary Fig. 4a, b). Combined with the 
a

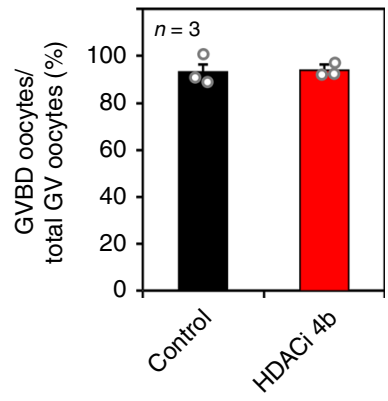

b

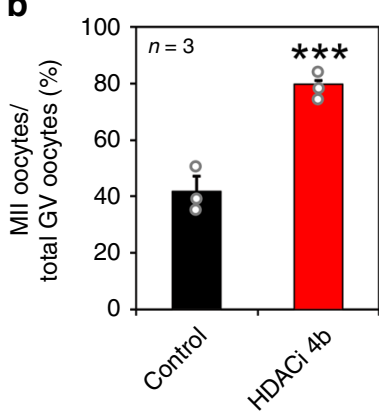

C

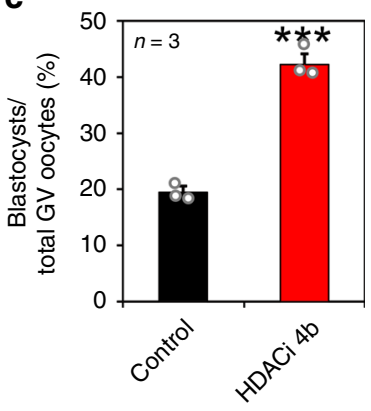

d
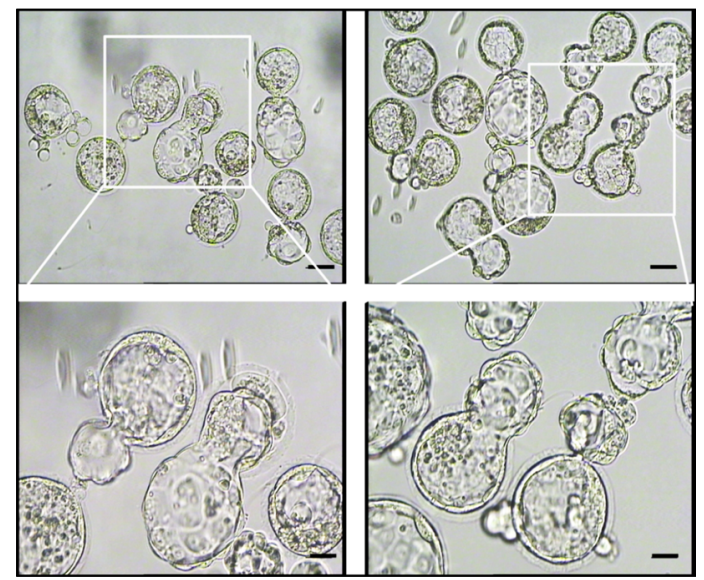

f

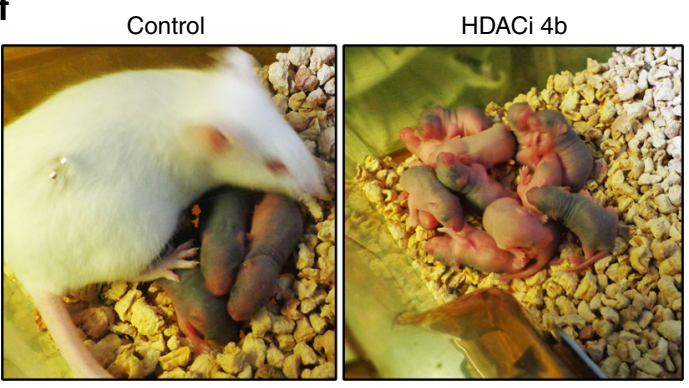

HDACi $4 b$

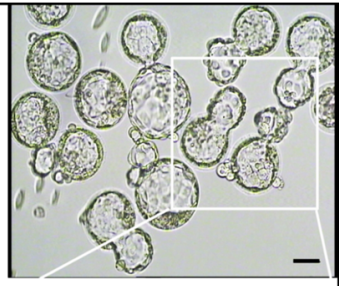

e
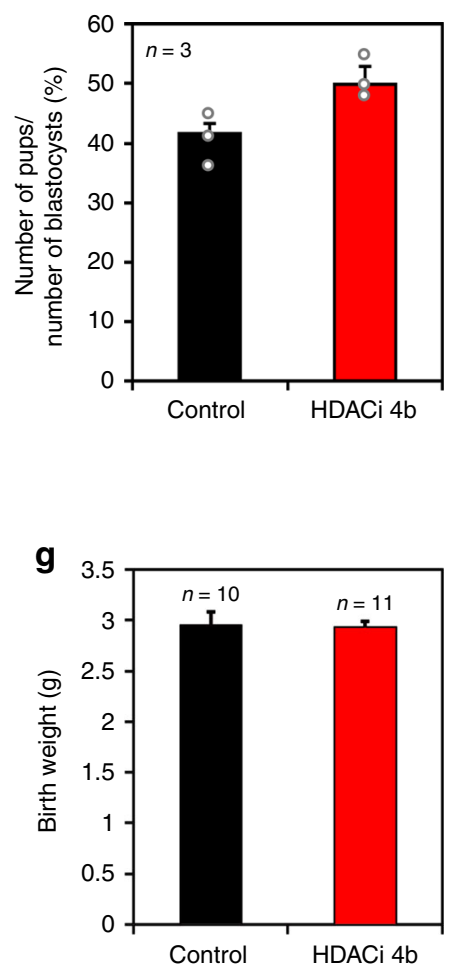

Fig. 6 The HDAC3 inhibitor improved mouse oocyte maturation and developmental competence. a The GVBD ratios were similar in the control and HDACi 4 b groups. HDACi 4b, HDAC3 inhibitor. b More MIl-stage oocytes were present in the HDACi $4 b$ group than in the control group. Data are presented as mean \pm SEM. Asterisks $\left(^{\star}\right)$ indicate significant differences at ${ }^{\star \star \star} P=0.0002$ determined by the two-sided $t$ test. $\mathbf{c}$ The blastocyst rate of all GV stage oocytes was considerably increased in the HDACi 4 b group compared with the control group. Data are presented as mean \pm SEM. Asterisks $(*$ ) indicate significant differences at ${ }^{\star \star \star} P=0.0006$ determined by the two-sided $t$ test. $\mathbf{d}$ Representative images show blastocysts obtained from the control and HDACi $4 \mathrm{~b}$ groups. Scale bars $=100 \mu \mathrm{m}$ and $40 \mu \mathrm{m}$ (enlarged). $n=3$ biologically independent experiments. e, f Blastocysts from the control and $\mathrm{HDACi} 4 \mathrm{~b}$ groups were transferred into surrogate mice. e Birth rates of the control and HDACi $4 \mathrm{~b}$ groups. $\mathbf{f}$ Representative images depicting the pups born in the control and HDACi $4 \mathrm{~b}$ groups. $\mathbf{g}$ The birth weights of the pups in the control and HDACi $4 \mathrm{~b}$ groups were similar. The data are presented as the mean \pm SEM. Source data are provided as a Source Data file.

result that the LH surge decreased HDAC3 levels in GCs and cumulus cells (Fig. 1a, b; Supplementary Fig. 1b, c), LH decreases HDAC3 protein levels in mGCs directly and in cumulus cells indirectly, resulting in increased AREG expression levels in both mGCs and cumulus cells and oocyte maturation. However, AREG may not be the sole target of HDACi 4 b; supplementing the IVM medium with AG1478, a specific inhibitor of the AREG receptor (EGFR), did not prevent 10-20\% of oocytes from maturing. Therefore, other unknown oocyte meiosis-related factors may be regulated by HDAC3 in GCs.

The reasons why altered expression of more genes was observed following treatment with the HDACi $4 \mathrm{~b}$ inhibitor compared with genetic loss of HDAC3 could be versatile. According to our study, the expression of Hdac3 in the ovarian GCs was only partially depleted whenever Hdac3 was deleted in $H d a c 3^{\text {CKO }}$ females by administration of tamoxifen because of the low efficiency of induced gene knockout. As one of the inhibitors of HDAC3, HDACi $4 \mathrm{~b}$ is also functional in inhibiting the activity of HDAC1. However, HDACi $4 \mathrm{~b}$ showed a $\sim 3-, \sim 25-$, and $\sim 72$-fold selectivity for HDAC3 over HDAC1, 2, and 8 respectively, and essentially no activity against other HDAC subfamily members ${ }^{18}$. The concentration of HDACi $4 \mathrm{~b}$ we used in this paper was the lowest effective concentration based on our preassays, which means that HDACi $4 \mathrm{~b}$ at this specific concentration 
could mimic the effect of HDAC3 depletion on AREG expression and oocyte maturation in vivo. In this study, more altered expression of genes was observed following HDACi $4 \mathrm{~b}$ treatment, instead of the in vivo group (Supplementary Fig. S6). Taking into account these above reasons, it is therefore understandable the differences between the data obtained in vivo resulting from HDAC3 partially depleted GCs and the data obtained in vitro resulting from HDAC3 fully inhibited GCs. Anyway, the expression of Areg in ovarian GCs was significantly increased in both $\mathrm{Hdac}^{\mathrm{cKO}}$ mice and HDACi $4 \mathrm{~b}$-treated GCs, which is in agreement with other results obtained in vitro and in vivo. In sum, Areg is an essential target gene of HDAC3 in mediating gonadotropin action on oocyte maturation.

Although HDACi $4 \mathrm{~b}$ preferentially targets HDAC3 and had limited in vitro and in vivo toxicity ${ }^{18}$, an evaluation of the safety of HDACi $4 \mathrm{~b}$ is still necessary before blastocysts produced with this system could be considered for practical use. Despite that $\mathrm{HDAC} 1 / 3$ belongs to the same class and directly interact in some cases, their cell type- and developmental stage-specific physiological actions may be distinct ${ }^{33}$. Interestingly, among all class I family HDACs currently identified in humans ${ }^{34}$, only HDAC3 responded to $\mathrm{LH}$ in preovulatory follicles in this study.

Physiologically, ovarian GCs mediates the action of gonadotropins on oocyte maturation and ovulation in the periovulatory follicles. Our results proved that, although the transcription factors (such as SP1) of AREG exists before LH surge in mGCs, these transcription factors cannot initiate AREG expression because the accessibility of Areg promoter is blocked by HDAC3. The findings emphasized the pivotal role of epigenetic modification of histone acetylation for the specifically open and closed of FSH and LH target genes in mGCs during oocyte maturation.

\begin{abstract}
Methods
Animal strains. C57BL/6J female mice were raised in colonies maintained by the investigators. Mice aged 21-23 days received an i.p. injection of 5 IU of PMSG (Sansheng Pharmaceutical Co., Ltd., Ningbo, China) to stimulate follicle growth. Forty-six to $48 \mathrm{~h}$ later, the mice received an i.p. injection of $5 \mathrm{IU}$ of hCG (Sansheng Pharmaceutical Co., Ltd., Ningbo, China) to induce oocyte maturation and ovulation. Hdac $3_{\text {flox/flox }}$ mice were purchased from the Jackson Laboratory (ME, USA), and Foxl2-CreER ${ }^{\mathrm{T} 2}$ mice were a gift from Dr. Liu Kui. All of these mice were housed under controlled temperature $\left(22^{\circ} \mathrm{C}\right)$ and light conditions $(14 \mathrm{~h}$ light, $10 \mathrm{~h}$ darkness; lights on at 07:00 a.m.) and allowed free access to chow and water. Briefly, to obtain the $\mathrm{Hdac}^{\mathrm{CKO}}$ mice, we crossed the Hdac $3^{\text {flox/flox }}$ females with Hdac $3_{\text {flox/flox }}$ plus Foxl2-CreER ER $^{\mathrm{T} 2}$ males without tamoxifen treatment. Therefore, the offsprings with $\mathrm{Hdac} 3^{\text {flox/flox }}$ but without Foxl2-CreER ${ }^{\mathrm{T} 2}$ were used as controls. The experiments were performed in accordance with the principles and guidelines for the use of laboratory animals of China Agricultural University and approved by the Institutional Animal Care and Use Committee of China Agricultural University.
\end{abstract}

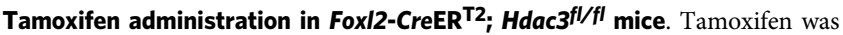
resuspended to a concentration of $100 \mathrm{mg} / \mathrm{mL}$ in $95 \%$ ethanol and further diluted with corn oil to a final concentration of $20 \mathrm{mg} / \mathrm{mL}$. A single i.p. injection of tamoxifen $(20 \mathrm{mg} / \mathrm{kg}$ body weight) was administered, respectively, to 18 -day-old Hdac $3^{\text {flox/flox }}$; Foxl2-CreER ${ }^{\mathrm{T} 2}$ female mice and $H d a c 3^{\text {flox/flox }}$ female mice (controls).

\section{Chemicals, hormones, and media. All chemicals were purchased from Sigma- Aldrich (St. Louis, MO, USA) unless otherwise noted. Media used for all experi- ments were obtained from Gibco (Life Technologies, CA, USA). All of the anti- bodies against histone acetylation were purchased from Active Motif (Shanghai, China) with the exception of the H3K14ac antibody, which was purchased from EpiGentek (Farmingdale, NY, USA). Other antibodies used in this paper are presented in Supplementary Table 3.}

Follicle, COC, and mGC culture. Follicles were isolated from the ovaries of PMSGprimed mice and cultured in M199 medium with ITS (insulin-transferrin-sodium selenite media supplement). To investigate the effect of the HDAC3 inhibitor HDACi 4b (sc207902, Santa Cruz Biotechnology, CA, USA) on oocyte maturation in vitro, the follicles were cultured for $16 \mathrm{~h}$ with or without $5 \mu \mathrm{M}$ HDACi $4 \mathrm{~b}$. Furthermore, to investigate the role of SP1 in AREG expression, $1 \mu \mathrm{M}$ MIT, an SP1 inhibitor, was added to both HDACi 4b-treated follicles and LH-treated follicles for
$4 \mathrm{~h}$. Gene expression in all these groups was examined at the end of the culture period.

Previous studies reported that PMSG was able to mimic the action of FSH in follicle development, and hCG mimicked the effects of LH on oocyte maturation and ovulation ${ }^{35-37}$. Therefore, PMSG and hCG are widely used as substitutes for FSH and LH in others' and our studies. Here COCs and mGCs were collected with a micropipette by puncturing large antral follicles with oocytes at the GV stage from the ovaries of mice that were pretreated with PMSG for $46-48 \mathrm{~h}$. The collected mGCs and COCs were maintained in hypoxanthine (HX)-IVM medium (M199 medium containing $4 \mathrm{mM} \mathrm{HX}, 75 \mathrm{~g} / \mathrm{mL}$ penicillin G, $50 \mathrm{~g} / \mathrm{mL}$ streptomycin sulfate, $0.29 \mathrm{mM}$ pyruvate, and $3 \mathrm{mg} / \mathrm{mL}$ bovine serum albumin (BSA)). To promote cell attachment, mGCs were cultured in IVM medium with $10 \%$ fetal bovine serum (FBS). Before COC culture, mGCs were treated with or without $5 \mu \mathrm{M}$ HDACi $4 \mathrm{~b}$ overnight to investigate the effect of mGC-derived HDAC 3 on oocyte maturation. Then COCs were added to the mGC feeders and cocultured for $16 \mathrm{~h}$ with $30 \mathrm{nM}$ NPPC. Furthermore, to elucidate whether AREG mediates the effects of HDAC 3 on oocyte maturation, $1 \mu \mathrm{M} \mathrm{AG1478}$, an EGFR inhibitor, was added to the mGC feeder medium. The GVBD ratio of oocytes in these COCs was analyzed. In addition, samples of mGCs and COCs were centrifuged, frozen, and stored at $-80^{\circ} \mathrm{C}$ for subsequent mRNA or protein expression analysis.

Follicles, oocytes, COCs, and mGCs were cultured at $37^{\circ} \mathrm{C}$ in a controlled atmosphere of $5 \% \mathrm{CO}_{2}$ and $95 \%$ air throughout the study.

RNA preparation and analysis. RNA was isolated from cultured cells and follicles using TRIzol Reagent (Invitrogen, Life Technologies, CA, USA) according to the manufacturer's protocol. The quantity and quality of total RNA were assessed using a Nanodrop spectrophotometer (Thermo Scientific, USA). Reverse transcription (RT) (Promega Reverse Transcription System) was performed using $1 \mu \mathrm{g}$ of total RNA per sample. qPCR was conducted and analyzed on an ABI 7500 Sequence Detection System (Applied Biosystems, USA) using a standard protocol The primers used for test genes are listed in Supplementary Table 1. For RNA-seq analysis of ovarian GCs from $H d a c 3^{\text {flox/flox }}$ and $H d a c 3^{\text {CKO }}$ mice, total RNA extracted from Hdac $3^{\text {flox/flox }}$ and $\mathrm{Hdac}^{\mathrm{CKO}}$ mouse ovaries was analyzed by Annoroad Gene Technology Co., Ltd. (Beijing, PR China). Furthermore, for singlecell RNA-seq analysis of oocytes cultured with or without HDACi 4 b, RNA was extracted from 35 oocytes per group, and transcriptome analysis was performed by Annoroad Gene Technology Co., Ltd. (Beijing, PR China).

Chromatin immunoprecipitation. To clarify whether HDAC3 directly binds to the Areg promoter, ChIP analysis was performed as described in the ChIP Assay Kit instructions (17-371, Millipore, MA, USA). In brief, cells extracted from mouse ovaries in vivo or cultured cells in vitro were cross-linked with $1 \%$ formaldehyde for $10 \mathrm{~min}$ at room temperature and neutralized by glycine at a final concentration of $0.125 \mathrm{M}$. After three washes with ice-cold phosphate-buffered saline (PBS), cell pellets were lysed in $1 \%$ sodium dodecyl sulfate (SDS) buffer containing a protease inhibitor. Then chromatin was sheared by sonication until the average DNA length was approximately $300-500 \mathrm{bp}$, as evaluated by $1 \%$ agarose gel electrophoresis. Sheared chromatin was diluted in ChIP dilution buffer to a final SDS concentration of $0.1 \%$. Then salmon sperm DNA/protein agarose slurry was added to preclear the chromatin solution. One percent of the chromatin fragment sample was stored at $4{ }^{\circ} \mathrm{C}$ for later use as nonprecipitated total chromatin (input) for normalization. The remaining chromatin fragments (99\%) were incubated overnight at $4{ }^{\circ} \mathrm{C}$ with $3 \mu \mathrm{g}$ of anti-HDAC3 antibody. Normal rabbit IgG (17-601, Millipore, MA, USA) was used as a negative control for nonspecific immunoprecipitation. The chromatin-antibody complex was incubated with protein A/G beads for $1 \mathrm{~h}$ at $4{ }^{\circ} \mathrm{C}$. The antibody/DNA complexes on agarose beads were collected by centrifugation. The beads were washed with various buffers in the following order low-salt immune complex buffer, high-salt immune complex buffer, LiCl immune complex buffer, and TE buffer provided by the supplier. Then the beads were suspended in elution buffer, and precipitated protein/DNA complexes were eluted from the antibodies/beads. The resulting protein/DNA complexes were then subjected to cross-link reversal in $0.2 \mathrm{M} \mathrm{NaCl}$ at $65^{\circ} \mathrm{C}$ for $10 \mathrm{~h}$, followed by the addition of $10 \mathrm{mM}$ EDTA, $40 \mathrm{mM}$ Tris- $\mathrm{HCl}$, and $40 \mu \mathrm{g} / \mathrm{ml}$ proteinase $\mathrm{K}$ and incubation at $55^{\circ} \mathrm{C}$ for $2 \mathrm{~h}$. DNA was purified by phenol/chloroform extraction and ethanol precipitation. Specific primers (Supplementary Table 2) were used to detect immunoprecipitated chromatin fragments and input chromatin.

Immunofluorescence. Ovaries and follicles were fixed in cold $4 \%$ paraformaldehyde (PFA) for 48 and $24 \mathrm{~h}$, respectively. Then the samples were dehydrated in ethanol and toluene, embedded in paraffin, and sectioned at 5- $\mu \mathrm{m}$ thickness. Ovaries and follicles were transferred to 3-aminopropyl-triethoxysilane-treated microscope slides (ZLI-9001, Zhongshan Company, Beijing, China) for immunofluorescence staining. In brief, sections were deparaffinized and rehydrated, and antigen retrieval was performed by microwaving for $15 \mathrm{~min}$ in $0.01 \%$ sodium citrate buffer (pH 6.0). For immunofluorescence analysis, sections were blocked with $10 \%$ normal donkey serum and incubated overnight at $4{ }^{\circ} \mathrm{C}$ with primary antibodies against HDAC3 before incubation with Alexa Fluor 488- or 555conjugated secondary antibodies (1:100, Invitrogen, Life Technologies, CA, USA) for $1 \mathrm{~h}$ at $37^{\circ} \mathrm{C}$ and propidium iodide (421301, BioLegend, CA, USA) or Hoechst 
33342 (B2261; Sigma-Aldrich, USA) as a nuclear counterstain. Samples were observed under a microscope.

Immunohistochemistry (IHC). For IHC, ovary sections were treated with 3\% $\mathrm{H}_{2} \mathrm{O}_{2}$ in $\mathrm{PBS}$ for $20 \mathrm{~min}$ to quench endogenous peroxidase activity. Nonspecific binding was blocked with 5\% BSA in PBS. Sections were then incubated overnight at $4{ }^{\circ} \mathrm{C}$ with the primary antibodies, incubated for $20 \mathrm{~min}$ at room temperature with horseradish peroxidase-labeled goat anti-rabbit IgG (Zhongshan Company, Beijing, China), and rinsed with PBS. The antibody complex was detected using $\mathrm{DAB}$ reagent according to the manufacturer's instructions (Zhongshan Company, Beijing, China).

Periodic acid Schiff (PAS) staining. To detect the corpora amylacea marker PAS, the sections were incubated in $0.5 \%$ periodic acid (Solarbio Company, Beijing, China) for $5 \mathrm{~min}$ at room temperature, followed by washing in tap water for $1 \mathrm{~min}$. Then the sections were incubated in Schiff reagent (Solarbio Company, Beijing, China) for $10 \mathrm{~min}$, followed by washing in tap water for $10 \mathrm{~min}$. After the sections were rinsed in PBS for $10 \mathrm{~min}$, the nuclei were stained with hematoxylin.

Coimmunoprecipitation. Fresh GCs were collected from the ovaries of 22-24-dayold female mice following stimulation with 5 IU of PMSG for $46 \mathrm{~h}$. Lysis buffer was added, and samples were centrifuged to obtain total protein. HDAC3 immunoprecipitation was performed according to the protocols of Dynabeads Protein G Immunoprecipitation Kit (Invitrogen, USA), as summarized below. Dynabeads were resuspended in Binding \& Washing buffer containing $5 \mu \mathrm{g}$ of anti-HDAC3 antibody and incubated for $40 \mathrm{~min}$ with rotation at room temperature. Dynabead-antibody complexes were then washed with Binding and Washing buffer. Then protein lysates were added for $1 \mathrm{~h}$ at room temperature with rotation, and the Dynabead-antibody-antigen complexes were washed thrice. Next, the complex was gently resuspended in $20 \mu \mathrm{L}$ of elution buffer and incubated for $5 \mathrm{~min}$ at $70^{\circ} \mathrm{C}$. The supernatant was transferred to a new tube and boiled in elution buffer for $10 \mathrm{~min}$ in a water bath at $100^{\circ} \mathrm{C}$. The nonspecific binding control was incubated with normal rabbit IgG (sc-2763, Santa Cruz Biotechnology, CA, USA). The supernatants of both the control and HDAC3 groups were subjected to liquid chromatography coupled with MS in the MS laboratory of China Agriculture University to identify all proteins that interacted with HDAC3.

Immunoblotting. Total protein was extracted in WIP (CellChip, BJ Biotechnology Co., Ltd., Beijing, China) as recommended by the manufacturer. In brief, total protein from each sample was separated by $10 \%$ SDS-polyacrylamide gel electrophoresis and then electrophoretically transferred onto polyvinylidene fluoride membranes (IPVH00010, Millipore, MA, USA). The membranes were then incubated for $1 \mathrm{~h}$ at room temperature with $5 \%$ BSA, followed by an overnight incubation at $4{ }^{\circ} \mathrm{C}$ with an anti-HDAC3 antibody. After being washed in TBST, the membranes were incubated with secondary antibody (1:5000, ZB-2301 and ZB-2305, ZSGB-BIO, Beijing, China) in TBST, followed by detection with the SuperSignal chemiluminescent detection system (34080, Thermo Scientific, CA, USA). Glyceraldehyde 3-phosphate dehydrogenase was used as an internal control.

DNA pulldown assay and bioinformatic analysis with JASPAR. To identify transcription factors that bind to the Areg promoter, DNA pulldown assays were performed as described previously ${ }^{38}$. Briefly, fresh GCs were collected from the ovaries of 22-24-day-old female mice stimulated with 5 IU of PMSG for $46 \mathrm{~h}$ and the nuclei were extracted from GCs with Buffer A (10 mM HEPES, pH 7.9, $1.5 \mathrm{mM}$ $\mathrm{MgCl}_{2}, 10 \mathrm{mM} \mathrm{KCl}, 300 \mathrm{mM}$ sucrose, and $\left.0.5 \% \mathrm{NP}-40\right)$. Nuclear protein was extracted from GC nucleus with NE (20 mM HEPES (pH7.9), $1.5 \mathrm{mM} \mathrm{MgCl}$, $0.5 \mathrm{M} \mathrm{NaCl}, 0.2 \mathrm{mM}$ EDTA and 20\% glycerol) and NET (Make up to $1 \%$ Triton-X100, $1 \mathrm{mM}$ dithiothreitol (DTT), $1 \mathrm{mM}$ phenylmethylsulfonyl fluoride in NE buffer) buffer as described ${ }^{38}$. Then primers targeting the Areg promoter ( -343 to -150 ) were synthesized with a $5^{\prime}$ biotin label, resulting in $5^{\prime}$-biotinylated doublestrand DNA probes. The DNA probes were incubated overnight with the extracted nuclear proteins. The DNA-protein complexes were incubated with streptavidinagarose beads (20347, Thermo Scientific, CA, USA) for $1 \mathrm{~h}$. The

DNA-protein-streptavidin bead complexes were washed three times with PBS. Finally, the complexes were eluted and subjected to MS analysis in the MS laboratory of China Agriculture University. For the bioinformatic analysis of potential transcription factor binding to the Areg promoter with JASPAR, we uploaded the Areg promoter sequence (from -343 to -150 ) onto the http://jaspar. genereg.net/ website.

Cross-linking Co-IP and reverse cross-linking Co-IP. To identify all the interactors of HDAC3 at chromatin, we performed cross-linking Co-IP assays as described previously ${ }^{24}$. Briefly, fresh GCs were collected from the ovaries of 22-24-day-old female mice stimulated with 5 IU of PMSG for $46 \mathrm{~h}$. Nuclear proteins were extracted from GCs with Buffer A (10 mM HEPES, pH 7.9, $1.5 \mathrm{mM}$ $\mathrm{MgCl}_{2}, 10 \mathrm{mM} \mathrm{KCl}, 300 \mathrm{mM}$ sucrose, and $0.5 \% \mathrm{NP}-40$ ) as described ${ }^{39}$. Then the nuclei were fixed with $1 \%$ formaldehyde diluted in PBS for $10 \mathrm{~min}$ at $37^{\circ} \mathrm{C}$. Crosslinked samples were quenched with glycine, washed once with PBS, and resuspended in $1 \mathrm{ml} \mathrm{NCB}(20 \mathrm{mM}$ Tris- $\mathrm{HCl} \mathrm{pH} 8,100 \mathrm{mM} \mathrm{KCl}, 5 \mathrm{mM} \mathrm{MgCl}, 10 \%$ glycerol, $0.1 \%$ NP-40, and $1 \mathrm{mM}$ DTT) supplemented with EDTA-free protein inhibitor cocktail (Millipore). Lysates were sonicated with a probe sonicator (Ningbo, China). Then the cross-linked chromatin extracts were cleared by centrifugation and subsequently treated with 1000 Kunitz units of Benzonase (Sigma) for $30 \mathrm{~min}$ at $30^{\circ} \mathrm{C}$. The extracts were immunoprecipitated overnight with antiHDAC3 magnetic beads (16-663, Millipore, MA, USA) or normal IgG magnetic beads. Then the complexes were washed three times and eluted with elution buffer for MS analysis in the MS laboratory of China Agriculture University. To confirm the interaction between FOXO1 and HDAC3, the cross-linking of the proteins eluted from the complexes was reversed by heating at $65^{\circ} \mathrm{C}$ overnight with $0.2 \mathrm{M}$ $\mathrm{NaCl}$. Then the reverse cross-linked proteins were analyzed by western blotting.

Oocyte IVM, IVF, and early embryo IVC. Four- to 5-week-old mice were used for this experiment. The mGCs retrieved from mice were cultured in Nunc IVF 4-well dishes (Thermo Scientific, Life Technologies, CA, USA) with IVM medium containing $10 \%$ FBS with or without $5 \mu \mathrm{M}$ HDAC3 inhibitor. The medium was covered with mineral oil (M8410, Sigma). The collected COCs were cocultured with mGC feeders. COCs in both groups were digested with hyaluronidase (90017, Vitrolife, Sweden) before examining meiosis progression. Oocytes at the MII stage in both groups were used for IVF. Mouse sperm capacitation was induced with IVF medium (Vitrolife, Sweden) for $1 \mathrm{~h}$. Then sperm were mixed with MII oocytes in IVF medium for $6 \mathrm{~h}$. Fertilization was confirmed by monitoring pronuclear formation. Then zygotes were transferred to G1 plus medium (Vitrolife, Sweden) and cultured until the embryos reached the eight-cell stage. Then zygotes were transferred into G2 plus medium (Vitrolife, Sweden) for further culture. The number of blastocysts was calculated and analyzed. The embryos were transferred into the uterus of a 2.5 -day postcoital pseudopregnant female mice as previously described ${ }^{38}$.

TUNEL. For TUNEL assays, ovaries were collected from Hdac $3^{\text {flox/flox }}$ and Hdac $3^{\mathrm{CKO}}$ mice and fixed in cold 4\% PFA for $1 \mathrm{~h}$. The tissues were embedded in paraffin wax and serially sectioned at $5 \mu \mathrm{m}$. Apoptotic cells were detected using a TUNEL Apoptosis Detection Kit (Invitrogen, Life Technologies, USA) after HDAC3 immunostaining.

Statistical analysis. All analysis were performed using Graph Pad Prism version 7 for Windows (GraphPad Software, La Jolla, CA, USA). Data for RT-PCR assays, follicle and oocyte counting experiments, and breeding tests are presented as mean \pm SEM, and each experiment was performed in triplicate. Data were analyzed using $t$ test or analysis of variance. Values of $P<0.05$ were considered statistically significant.

Reporting summary. Further information on research design is available in the Nature Research Reporting Summary linked to this article.

\section{Data availability}

RNA-seq data have been submitted to the NCBI Gene Expression Omnibus (GEO; http://www.ncbi.nlm.nih.gov/geo/) under accession number GSE139666 for the SuperSeries record of the three groups of RNA-seq data, including GSE139663, GSE139664, and GSE139665, respectively, for the transcriptome of HDAC3 ${ }^{\mathrm{CKO}}$ mice ovarian mGCs, the cultured ovarian mGCs treated with HDACi $4 \mathrm{~b}$, and the cultured oocytes treated with HDACi 4b. Proteomics data of Fig. 4 are available in Supplementary Data $4-8$. The reporting summary and editorial checklist for this article are available as a Supplementary file.

Received: 6 November 2018; Accepted: 18 November 2019; Published online: 16 December 2019

\section{References}

1. Hsieh, M. et al. Luteinizing hormone-dependent activation of the epidermal growth factor network is essential for ovulation. Mol. Cell. Biol. 27, 1914-1924 (2007)

2. Conti, M., Hsieh, M., Zamah, A. M. \& Oh, J. S. Novel signaling mechanisms in the ovary during oocyte maturation and ovulation. Mol. Cell. Endocrinol. 356, 65-73 (2012).

3. Zhang, M., Su, Y. Q., Sugiura, K., Xia, G. \& Eppig, J. J. Granulosa cell ligand NPPC and its receptor NPR2 maintain meiotic arrest in mouse oocytes. Science 330, 366-369 (2010)

4. Richard, F. J., Tsafriri, A. \& Conti, M. Role of phosphodiesterase type 3A in rat oocyte maturation. Biol. Reprod. 65, 1444-1451 (2001).

5. Vaccari, S., Weeks, J. L. 2nd, Hsieh, M., Menniti, F. S. \& Conti, M. Cyclic GMP signaling is involved in the luteinizing hormone-dependent meiotic maturation of mouse oocytes. Biol. Reprod. 81, 595-604 (2009). 
6. Park, J. Y. et al. EGF-like growth factors as mediators of LH action in the ovulatory follicle. Science 303, 682-684 (2004).

7. Wang, X. et al. High level of C-type natriuretic peptide induced by hyperandrogen-mediated anovulation in polycystic ovary syndrome mice. Clin. Sci. 132, 759-776 (2018).

8. Liu, W. et al. Estrogen receptors in granulosa cells govern meiotic resumption of pre-ovulatory oocytes in mammals. Cell Death Dis. 8, e2662 (2017).

9. Zhang, M. \& Xia, G. Hormonal control of mammalian oocyte meiosis at diplotene stage. Cell. Mol. Life Sci. 69, 1279-1288 (2012).

10. Fang, J. et al. Involvement of protein acyltransferase ZDHHC3 in maintaining oocyte meiotic arrest in Xenopus laevis. Biol. Reprod. 95, 67 (2016). 61-69-67, 61-69.

11. Yoshida, N., Brahmajosyula, M., Shoji, S., Amanai, M. \& Perry, A. C. Epigenetic discrimination by mouse metaphase II oocytes mediates asymmetric chromatin remodeling independently of meiotic exit. Dev. Biol. 301, 464-477 (2007)

12. Lee, L. et al. Changes in histone modification and DNA methylation of the StAR and Cyp19al promoter regions in granulosa cells undergoing luteinization during ovulation in rats. Endocrinology 154, 458-470 (2013).

13. Strahl, B. D. \& Allis, C. D. The language of covalent histone modifications. Nature 403, 41-45 (2000).

14. Qu, F. et al. A molecular mechanism underlying ovarian dysfunction of polycystic ovary syndrome: hyperandrogenism induces epigenetic alterations in the granulosa cells. J. Mol. Med. 90, 911-923 (2012).

15. Grunstein, M. Histone acetylation in chromatin structure and transcription. Nature 389, 349-352 (1997).

16. Alenghat, T. et al. Histone deacetylase 3 coordinates commensal-bacteriadependent intestinal homeostasis. Nature 504, 153-157 (2013).

17. Zheng, W. et al. Two classes of ovarian primordial follicles exhibit distinct developmental dynamics and physiological functions. Hum. Mol. Genet. 23 920-928 (2014).

18. Jia, H. et al. Histone deacetylase (HDAC) inhibitors targeting HDAC3 and HDAC1 ameliorate polyglutamine-elicited phenotypes in model systems of Huntington's disease. Neurobiol. Dis. 46, 351-361 (2012).

19. Hernandez-Gonzalez, I. et al. Gene expression profiles of cumulus cell oocyte complexes during ovulation reveal cumulus cells express neuronal and immune-related genes: does this expand their role in the ovulation process? Mol. Endocrinol. 20, 1300-1321 (2006).

20. Wang, Y. et al. Epidermal growth factor receptor signaling-dependent calcium elevation in cumulus cells is required for NPR2 inhibition and meiotic resumption in mouse oocytes. Endocrinology 154, 3401-3409 (2013).

21. Karagianni, P. \& Wong, J. HDAC3: taking the SMRT-N-CoRrect road to repression. Oncogene 26, 5439-5449 (2007).

22. Guenther, M. G., Barak, O. \& Lazar, M. A. The SMRT and N-CoR corepressors are activating cofactors for histone deacetylase 3. Mol. Cell. Biol. 21, 6091-6101 (2001).

23. Watson, P. J., Fairall, L., Santos, G. M. \& Schwabe, J. W. Structure of HDAC3 bound to co-repressor and inositol tetraphosphate. Nature 481, 335-340 (2012).

24. Armour, S. M. et al. An HDAC3-PROX1 corepressor module acts on HNF4alpha to control hepatic triglycerides. Nat. Commun. 8, 549 (2017).

25. Bonnet, A., Dalbiès-Tran, R. \& Sirard, M. A. Opportunities and challenges in applying genomics to the study of oogenesis and folliculogenesis in farm animals. Reproduction 135, 119-128 (2008).

26. Zamah, A. M. et al. Human oocyte maturation is dependent on LH-stimulated accumulation of the epidermal growth factor-like growth factor, amphiregulin. Hum. Reprod. 25, 2569-2578 (2010).

27. Conti, M. et al. Role of cyclic nucleotide signaling in oocyte maturation. Mol. Cell. Endocrinol. 187, 153-159 (2002).

28. Jurema, M. W. \& Nogueira, D. In vitro maturation of human oocytes for assisted reproduction. Fertil. Steril. 86, 1277-1291 (2006).

29. Feng, R. et al. Mutations in TUBB8 and human oocyte meiotic arrest. N. Engl. J. Med. 374, 223-232 (2016).

30. Swain, J. E. \& Pool, T. B. ART failure: oocyte contributions to unsuccessful fertilization. Hum. Reprod. Update 14, 431-446 (2008).

31. Chen, J. et al. Somatic cells regulate maternal mRNA translation and developmental competence of mouse oocytes. Nat. Cell Biol. 15, 1415-1423 (2013).

32. Santos, M. A., Kuijk, E. W. \& Macklon, N. S. The impact of ovarian stimulation for IVF on the developing embryo. Reproduction 139, 23-34 (2010).

33. Thomas, E. A. et al. The HDAC inhibitor $4 \mathrm{~b}$ ameliorates the disease phenotype and transcriptional abnormalities in Huntington's disease transgenic mice. Proc. Natl Acad. Sci. USA 105, 15564-15569 (2008).
34. Xu, W. S., Parmigiani, R. B. \& Marks, P. A. Histone deacetylase inhibitors: molecular mechanisms of action. Oncogene 26, 5541-5552 (2007).

35. Popova, E., Krivokharchenko, A., Ganten, D. \& Bader, M. Comparison between PMSG- and FSH-induced superovulation for the generation of transgenic rats. Mol. Reprod. Dev. 63, 177-182 (2002).

36. Pramoda, S. \& Saidapur, S. K. Preponement of ovarian follicular development in the frog, Rana tigerina, using PMSG, HCG, growth hormone, heteroplastic pituitary pars distalis homogenate, FSH and LH. Indian J. Exp. Biol. 22, 527-532 (1984)

37. Neal, P. \& Baker, T. G. Response of mouse ovaries in vivo and in organ culture to pregnant mare's serum gonadotrophin and human chorionic gonadotrophin. 3. Effect of age. J. Reprod. Fertil. 39, 411-414 (1974).

38. Singer, O., Tiscornia, G., Ikawa, M. \& Verma, I. M. Rapid generation of knockdown transgenic mice by silencing lentiviral vectors. Nat. Protoc. 1, 286-292 (2006).

39. Wu, K. K. in Gene Mapping, Discovery, and Expression: Methods and Protocols (ed. Bina, M.) 281-290 (Humana Press, Totowa, NJ, 2006).

\section{Acknowledgements}

We are indebted to Dr. Marco Conti (Center for Reproductive Sciences at University of California, San Francisco) for valuable suggestions to optimize the experimental design. This work was supported by grants from the National Key Research \& Developmental Program of China (2018YFC1003800 to C.W.; 2018YFC1003700 and 2017YFC1001100 to G.X.); the National Basic Research Program of China (2013CB945500 to G.X., 2014CB943202 to C.W.); National Natural Science Foundation of China (31872792 to C.W., 31371448 and 31571540 to G.X.); Beijing Natural Science Foundation (5182015 to C.W., 7182090 to G.X.); Chinese Universities Scientific Fund (2015QC018 to C.W.); Project of State Key Laboratory of Agrobiotechnology (2015SKLAB4-1 to C.W. and 2016SKLAB-1 to G.X.); and Institution of Higher Education Projects of Building Firstclass Discipline Construction in Ningxia Region (Biology) (NXYLXK2017B05) to G.X.

\section{Author contributions}

Hu.W., H.C., G.X., and C.W. designed the work. Hu.W., H.C., and X.W. performed and analyzed the majority of the experiments. M.Z., B.L., Z.C., T.Y., J.F., Y.Z., W.L., J.H., and Q.G. analyzed the data and contributed to reagents, materials, or analysis tools. H.Z and Ha.W. provided their helpful comments during preparation of this paper. The manuscript was written by Hu.W. and H.C. and revised by G.X. and C.W.

\section{Competing interests}

The authors declare no competing interests.

\section{Additional information}

Supplementary information is available for this paper at https://doi.org/10.1038/s41467019-13671-8.

Correspondence and requests for materials should be addressed to C.W.

Peer review information Nature Communications thanks David Albertini, Kui Liu, and the other anonymous reviewer(s) for their contribution to the peer review of this work

Reprints and permission information is available at http://www.nature.com/reprints

Publisher's note Springer Nature remains neutral with regard to jurisdictional claims in published maps and institutional affiliations.

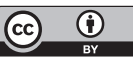

Open Access This article is licensed under a Creative Commons Attribution 4.0 International License, which permits use, sharing, adaptation, distribution and reproduction in any medium or format, as long as you give appropriate credit to the original author(s) and the source, provide a link to the Creative Commons license, and indicate if changes were made. The images or other third party material in this article are included in the article's Creative Commons license, unless indicated otherwise in a credit line to the material. If material is not included in the article's Creative Commons license and your intended use is not permitted by statutory regulation or exceeds the permitted use, you will need to obtain permission directly from the copyright holder. To view a copy of this license, visit http://creativecommons.org/ licenses/by/4.0/.

(C) The Author(s) 2019 